\title{
Geometric rigidity of conformal matrices
}

\author{
DANIEL FARACO AND XIAO ZHONG
}

\begin{abstract}
We provide a geometric rigidity estimate à la Friesecke-James-Müller for conformal matrices. Namely, we replace $\mathrm{SO}(n)$ by an arbitrary compact set of conformal matrices, bounded away from 0 and invariant under $\mathrm{SO}(n)$, and rigid motions by Möbius transformations.
\end{abstract}

Mathematics Subject Classification (2000): 30C65 (primary); 49J45 (secondary).

\section{Introduction}

This paper is concerned with the so-called geometric rigidity estimates for conformal matrices. Recently, Friesecke, James and Müller developed a successful new approach to the classical problem of dimension-reduction in nonlinear elasticity $[7,8]$. A fundamental ingredient was the following rigidity estimate for the group $\mathrm{SO}(n)=\left\{A \in M^{n \times n}: A^{t} A=I, \operatorname{det} A=1\right\}$ of special orthogonal matrices in $\mathbb{R}^{n}$.

Theorem 1.1. Let $\Omega \subset \mathbb{R}^{n}$ be a bounded Lipschitz domain and $n \geq 2$. There exists a constant $C_{1}=C_{1}(\Omega)$ with the property that for each $v \in W^{1,2}\left(\Omega, \mathbb{R}^{n}\right)$, there exists $R \in \mathrm{SO}(n)$ such that

$$
\|D v-R\|_{L^{2}(\Omega)} \leq C_{1}\left\|\operatorname{dist}_{\mathrm{SO}(n)}(D v)\right\|_{L^{2}(\Omega)} .
$$

Theorem 1.1 has been used in a number of related problems concerning dimensionreduction, e.g. [3, 6, 18] and [17]. In all the applications it is crucial that the dependence between the left- and right-hand side is linear and that $v$ is any general Sobolev mapping (the classical result of John [15] gives an $L^{2}-L^{\infty}$ estimate valid for locally bi-Lipschitz maps). Theorem 1.1 makes quantitative the following classical result of Reshetnyak [19] for sequences.

Theorem 1.2. Let $\left\{v_{j}\right\} \in W^{1,2}(\Omega)$ be a weakly convergent sequence in $W^{1,2}$. Then there exists $R \in \mathrm{SO}(n)$ such that

$$
\lim _{j \rightarrow \infty}\left\|\operatorname{dist}_{\mathrm{SO}(n)}\left(D v_{j}\right)\right\|_{L^{2}(\Omega)}=0 \Rightarrow \lim _{j \rightarrow \infty}\left\|D v_{j}-R\right\|_{L^{2}(\Omega)}=0 .
$$


A natural question raised by Theorem 1.1 is to determine when a qualitative rigidity theorem like Theorem $1.2 \mathrm{can}$ in fact be made quantitative in the sense of Theorem 1.1. That is, let $E \subset M^{n \times n}$ be such that for a weakly convergent sequence $\left\{v_{j}\right\} \in W^{1,2}(\Omega)$ it holds that

$$
\lim _{j \rightarrow \infty}\left\|\operatorname{dist}_{E}\left(D v_{j}\right)\right\|_{L^{2}(\Omega)}=0 \Rightarrow \lim _{j \rightarrow \infty}\left\|D v_{j}-D \varphi\right\|_{L^{2}(\Omega)}=0
$$

where $\varphi \in W^{1,2}\left(\Omega, \mathbb{R}^{n}\right)$ is such that $D \varphi \in E$ a.e.

Then, we would like to know if there exists a constant $C(\Omega)$ such that for every $v \in W^{1,2}\left(\Omega, \mathbb{R}^{n}\right)$ there exists $\varphi$ with $D \varphi \in E$ and

$$
\|D v-D \varphi\|_{L^{2}(\Omega)} \leq C(\Omega)\left\|\operatorname{dist}_{E}(D v)\right\|_{L^{2}(\Omega)} .
$$

In particular, since Reshetnyak proved results related to Theorem 1.2 for subsets of the set of conformal matrices

$$
\mathrm{CO}_{+}(n)=\left\{A \in M^{n \times n}: A=\rho R, \text { where } \rho \in \mathbb{R}_{+} \text {and } R \in \mathrm{SO}(n)\right\},
$$

in [8] it was posed the question whether his results can made quantitative in the sense of Theorem 1.1.

In this work we show that if $E$ is a compact subset of $\mathrm{CO}_{+}(n)$, invariant under $\mathrm{SO}(n)$ and with $0 \notin E$, a quantitative rigidity estimate holds (see Theorem 1.4). Before stating the result, we need to recall that for $E \Subset \mathrm{CO}_{+}(n)$ the solutions of the differential inclusion

$$
D \varphi \in E, \quad \varphi \in W^{1,2}(\Omega)
$$

are described by the Liouville Theorem.

Theorem 1.3 (Liouville Theorem). Let $\Omega \subset \mathbb{R}^{n}, n \geq 3$ and let $\varphi \in W^{1, n}\left(\Omega, \mathbb{R}^{n}\right)$ be such that

$$
D \varphi(x) \in \mathrm{CO}_{+}(n) \text { a.e. } x \in \Omega \text {. }
$$

Then

$$
\varphi(x)=b+A x, \operatorname{or} \varphi(x)=b+A R \frac{x-a}{|x-a|^{2}}
$$

where $b \in \mathbb{R}^{n}, a \in \mathbb{R}^{n} \backslash \Omega, A \in \mathrm{CO}_{+}(n)$ and $R=\operatorname{diag}(1, \ldots,-1)$.

The Liouville Theorem has a long history. Liouville established it for $C^{3}$ mappings in [16], Gehring for homeomorphisms in $W^{1, n}\left(\Omega, \mathbb{R}^{n}\right)$ in [9] and Reshetnyak removed the injectivity assumption in [19]. In fact, $W^{1, n}$ is not the borderline case. Iwaniec [13] proved that there exists a critical threshold $p_{n}<n$ such that Liouville theorem holds for mappings in $W^{1, p}\left(\Omega, \mathbb{R}^{n}\right), p \geq p_{n}$. Moreover, Iwaniec and 
Martin [14] proved that if $n=2 m$ and $m$ is an integer then $p_{n}=m$ and the result is optimal. For $n$ odd $p_{n}$ is conjectured to be also $\frac{n}{2}$.

Geometrically, the Liouville theorem relates $\mathrm{CO}_{+}(n)$ with the special Möbius group $\mathcal{M}_{n}$. The Möbius group $\mathbf{M o ̈ b}(n)$ is the group generated by reflections on spheres and hyperplanes. Then $\mathcal{M}_{n}$ consists of Möbius transformations preserving orientation (see [1] for an introduction of the geometry of the Möbius group and its discrete subgroups). It turns out that any Möbius transform can be represented as the composition of an affine mapping and an inversion with respect to a sphere. Analytically, this yields formula (1.7).

We are interested in compact subsets of $\mathrm{CO}_{+}(n)$ invariant under $\mathrm{SO}(n)$. For technical reasons we also assume that these sets have finitely many connected components. Let us introduce the notation

$$
\begin{array}{r}
E^{m, M}=\left(\mathrm{CO}_{+}(n) \cap B(0, M)\right) \backslash B(0, m), \\
m \mathrm{SO}(n)=\{m R: R \in \mathrm{SO}(n)\} .
\end{array}
$$

Then our conditions on $E$ imply that it can be represented by,

$$
E=\cup_{i=1}^{n_{1}} E^{m_{i}, M_{i}} \cup_{i=1}^{n_{2}} m_{i} \mathrm{SO}(n) .
$$

In addition, since the sets $E$ are compact the solutions to (1.5) are in particular Möbius transforms. We denote them by $\mathcal{M}_{n}^{E}(\Omega)$. An interesting new feature respect to the other nonlinear sets for which quantitative rigidity estimates are available (e.g. [2]) is that $\mathcal{M}_{n}^{E}(\Omega)$ contains non affine solutions. We are now in the position to state the rigidity estimate:

Theorem 1.4. Let $E \subset \mathrm{CO}_{+}(n)$ be compact, finitely connected, with $0 \notin E$ and such that

$$
\mathrm{SO}(n) E=E
$$

Let $\Omega^{\prime} \Subset \Omega \subset \mathbb{R}^{n}, n \geq 3$ and $\Omega$ be a bounded domain. Then,

i) There exists a constant $C_{2}=C_{2}\left(E, \Omega^{\prime}, \Omega\right)$ such that for any $v \in W^{1,2}\left(\Omega, \mathbb{R}^{n}\right)$ there exists $\varphi \in \mathcal{M}_{n}^{E}\left(\Omega^{\prime}, \mathbb{R}^{n}\right)$ such that

$$
\int_{\Omega^{\prime}}|D \varphi-D v|^{2} \leq C_{2} \int_{\Omega} \operatorname{dist}_{E}^{2}(D v) .
$$

ii) Let $\Omega$ be Lipschitz. Then, a constant $C_{3}=C_{3}(E, \Omega)$ such that

$$
\int_{\Omega}|D \varphi-D v|^{2} \leq C_{3} \int_{\Omega} \operatorname{dist}_{E}^{2}(D v)
$$

exists if and only if $E=\cup_{i=1}^{n_{3}} m_{i} \mathrm{SO}(n)$. 
We do not know if we could put $E=\mathrm{CO}_{+}(n)$ in Theorem 1.4. However if we restrict our attention to mappings of finite distortion with suitable integrability conditions on the distortion function a rigidity estimate holds, as it will be shown elsewhere. The proof relies heavily on Theorem 1.4 and a recent result of Koskela, Hencl, and Zhong [12] about the doubling properties of the Jacobian of this kind of mappings. The case $n=2$ is very different. It holds that $\mathrm{CO}_{+}(2)$ is a linear subspace and by Weyl's lemma $W^{1,1}$ solutions to the differential inclusion

$$
D \varphi \in \mathrm{CO}_{+}(2)
$$

are holomorphic functions. Then the rigidity estimate for $E=\mathrm{CO}_{+}(2)$ holds (for every exponent $1<p \leq \infty)$ by the boundedness of the Ahlfors-Beurling transform (for a proof see [5]). However, since holomorphic function are not as rigid as Möbious transforms, it remains open what is the situation for the corresponding set $E^{M, m}$ in two dimensions.

Our approach to Theorem 1.4 is essentially a combination of ideas of [8] with those developed by Reshetnyak in his study of the stability of Liouville theorem respect to different parameters and classes of functions (See [21], in particular Theorems 3.2 and 5.2). The paper is organized as follows. In Section 2 we state some basic facts about Möbius mappings and solutions to elliptic equations. Section 3 is devoted to prove that arbitrary Möbious transforms are approximated in the sense of Theorem 1.4 by mappings in $\mathcal{M}_{n}^{E}(\Omega)$. In this section, we provide an example showing that, if $\mathcal{M}_{n}^{E}(\Omega)$ contains non affine mappings, a global estimate like in Theorem 1.4 (ii) does not hold even in this simplified setting.

Section 4 constitutes the crux of the paper. We prove Theorem 1.4 for $\Omega^{\prime}$ a ball. The proof is based on the fact that $E$ is related to elliptic equations globally and locally. Globally, there exists a smooth uniformly convex mapping $F: \mathbb{R}^{n} \rightarrow \mathbb{R}$ such that for every $A \in E$

$$
F\left(A_{i}\right)=\operatorname{det}(A),
$$

where $A_{i}$ is any row of the matrix $A$. The existence of such a mapping $F$ enable us to write

$$
v=w+z
$$

where each of the coordinates $z_{i}$ satisfies the equation

$$
\operatorname{div}\left(D F\left(D z_{i}\right)\right)=0,
$$

and $\int_{\Omega}|\nabla w|^{2} \leq C \int_{\Omega} \operatorname{dist}_{E}^{2}(D v)$. Hence, it suffices to prove Theorem 1.4 for such a mapping $z$. Next, the regularity theory of elliptic equations implies that $z_{i}$ enjoys a priori estimates. In particular, the modulus of continuity of $D z$ is uniformly bounded. This allows the use of a compactness argument to deduce that it is essentially enough to prove Theorem 1.4 for mappings $z$ such that

$$
\|\operatorname{dist}(D z, I)\|_{L^{\infty}\left(\Omega^{\prime}\right)}<<1
$$


and the modulus of continuity of $D z$ is uniformly bounded. In this situation we can use the local equation which is given by the tangent plane to $\mathrm{CO}_{+}(n)$. We proceed by adapting the ideas of Reshetnyak [21] to our situation. Beside a Korn type inequality for the tangent plane to $\mathrm{CO}_{+}(n)$, a degree argument involving the exponential map of $\mathcal{M}_{n}$ is needed to choose the Möbius mapping closest to $z$. In this way, one obtains a mapping $\varphi^{\prime} \in \mathcal{M}_{n}\left(\Omega^{\prime}\right)$ satisfying (1.10). However, these arguments do not imply that $\varphi^{\prime} \in \mathcal{M}_{n}^{E}\left(\Omega^{\prime}\right)$. The desired mapping $\varphi$ is obtained by applying section 3 to $\varphi^{\prime}$. Let us remark that this is the only moment along the whole proof where the $\mathrm{SO}(n)$ invariance of $E$ is used. The last section is devoted to prove Theorem 1.4 for an arbitrary $\Omega^{\prime} \Subset \Omega$.

ACKnowledgement. We thank Stefan Müller for bringing the problem to our attention and for many interesting discussions and suggestions. Part of this research took part when D. F. was visiting Wuhan Institute of Physics and Mathematics, and when X. Z. was visiting the Max-Planck Institute for Mathematics in the Sciences. We would like thank the hospitality and research environment in both institutions. This work was partially supported by the EU Research Training Networks HYperbolic and Kinetic Equations, contract HPRN-CT-2002-00282 and Phase Transitions in crystalline solids, contract FMRX-CT 98-0229.

\section{Notation and preliminaries}

We will denote by $C_{1}, C_{2}, \ldots, C_{n}$ constants which will be used during the whole paper, whereas $c_{1}, c_{2}, \ldots, c_{n}$ will be used for different constants within the same proof.

Concerning sets in $\mathbb{R}^{n}$, we will use $B$ for balls and $B(a, r)$ where we want to specify the center $a$ and the radius $r$. For balls centered at the origin we use $B_{r}=B(0, r)$. Given a ball $B(a, r), h B=B(a, h r)$. For a measurable set $L,|L|$ denotes its Lebesgue measure. Let $K \subset \mathbb{R}^{n}$ and $s \in \mathbb{R}$. Then $K_{s}=\left\{y \in \mathbb{R}^{n}\right.$ : $\operatorname{dist}(y, K) \leq s\}$.

Let $A=\left(a_{i j}\right) \in M^{n \times n}$ then $|A|$ stands for the operator norm. Given a closed set $E \in M^{n \times n} \operatorname{dist}(A)=\inf _{B \in E}|A-B|$. Let us remark the choice of the operator norm in our definition of the distance is motivated to simplify the constants in section 3 but of course any other norm would do.

Let $E$ be the set in Theorem 1.4. Since $0 \notin E$ and $E$ is compact there exists $0<m<M<\infty$ such that

$$
E \subset E^{m, M}
$$

where $E^{m, M}$ was introduced in (1.9).

We will used the notation $E^{\prime}$ for an auxiliary set different $E$ but satisfying the assumptions of Theorem 1.4. In particular it will satisfy (2.1) for some other numbers $m^{\prime}$ and $M^{\prime}$. 
Given a closed set $\Omega \subset \mathbb{R}^{n}, \mathcal{M}_{n}(\Omega)$ are Möbius transforms which are finite in $\Omega, \mathcal{M}_{n}\left(\Omega_{1}, \Omega_{2}\right)$ stands for Möbius transform mapping $\Omega_{1}$ onto $\Omega_{2}$. Let us recall the notation

$$
\mathcal{M}_{n}^{E}(\Omega)=\left\{\varphi \in W^{1,2}(\Omega): D \varphi(x) \in E \text { a.e. } x \in \Omega\right\} .
$$

We discuss now several basic properties of $\mathcal{M}_{n}(\Omega)$ and more precisely of $\mathcal{M}_{n}^{E}(B)$. In particular we show in the first lemma that mappings $\varphi \in \mathcal{M}_{n}^{E}(B)$ can be handled in an uniform way. The main reason is that for $\varphi \in \mathcal{M}_{n}^{E}(B) \varphi^{-1}(\infty)$, the center of the sphere associated to $\varphi$, is bounded away from $B$ independently of $\varphi$. For the notation, recall that for $\varphi \in \mathcal{M}_{n}, \varphi(B)$ is a ball.

Lemma 2.1. Let $\varphi \in \mathcal{M}_{n}^{E}(B)$ and let $m, M$ be the constants in (2.1). Then the following three properties hold:

(1) $m^{n} \leq \frac{|\varphi(B)|}{|B|} \leq M^{n}$.

(2) $\varphi \in \mathcal{M}_{n}\left(h_{0} B\right)$ where $h_{0}=h_{0}\left(\frac{M}{m}\right)>1$.

(3) Let $s<1$. Then there exists a number $h_{1}=h_{1}(s, E)<1$ such that $\varphi(s B) \subset$ $h_{1} \varphi(B)$ and $s \varphi(B) \subset \varphi\left(h_{1} B\right)$.

Proof. Since for $A \in \mathrm{CO}_{+}(n),|A|^{n}=\operatorname{det}(A)$, it follows that

$$
|\varphi(B)|=\int_{B} J_{\varphi}=\int_{B}|D \varphi|^{n}
$$

(1) follows from (2.1).

We prove (2) for $\varphi \in \mathcal{M}_{n}^{E}(B)$ not affine. It follows from (1.7) that for such $\varphi$ there exists $r \in \mathbb{R}$ and $a \in \mathbb{R}^{n}$. such that $|D \varphi(x)|=r^{2}|x-a|^{-2}$. Thus, $\max _{B}|D \varphi|=r^{2} \operatorname{dist}_{B}(a)^{-2}$ and $\min _{B}|D \varphi|=r^{2}\left(\operatorname{dist}_{B}(a)+\operatorname{diam}(B)\right)^{-2}$. Since $\varphi \in \mathcal{M}_{n}^{E}(B)$ we have that

$$
\frac{r^{2} \operatorname{dist}_{B}(a)^{-2}}{r^{2}\left(\operatorname{dist}_{B}(a)+\operatorname{diam}(B)\right)^{-2}} \leq \frac{M}{m},
$$

i.e.

$$
\operatorname{dist}_{B}(a) \geq \frac{\operatorname{diam}(B)}{\sqrt{\frac{M}{m}}-1} .
$$

Hence $h_{0}=1+2 \frac{1}{\sqrt{\frac{M}{m}}-1}$.

For (3), we firstly observe there is no loss of generality in assuming that $B=$ $B_{1}$ and $\varphi\left(B_{1}\right)=B_{1}$. The general case follows by considering similarities $T_{B}, T_{\varphi(B)}$ such that $T_{B}\left(B_{1}\right)=B$ and $T_{\varphi(B)}\left(B_{1}\right)=\varphi(B)$. Then the mapping $\tilde{\varphi}=T_{\varphi(B)}^{-1} \circ \varphi \circ T_{B}$ 
satisfies that $\tilde{\varphi}\left(B_{1}\right)=B_{1}$ and $m^{2} \leq|D \tilde{\varphi}| \leq M^{2}$. It is easy to check that if the thesis holds for $\tilde{\varphi}$ it also holds for $\varphi$.

Therefore there is no loss of generality assuming $\varphi \in \mathcal{M}_{n}^{E}\left(B_{1}, B_{1}\right)$. Since $\frac{1}{M} \leq\left|D \varphi^{-1}\right| \leq \frac{1}{m}$, (2) implies that $\varphi^{-1} \in \mathcal{M}_{n}\left(h_{0} B_{1}\right)$ for the same $h_{0}$. Thus, $\varphi(\infty)=b$, with $|b| \geq h_{0}$.

On the other hand, $\varphi\left(B_{1}\right)=B_{1}$ implies that $\varphi$ fixes $S^{n-1}$. Thus, it conjugates with the mapping $R=\frac{x}{|x|^{2}}$ (see [1, Theorem 3.2.4]), i.e. $\varphi(x)=R \circ \varphi \circ R(x)$. Putting $x=0$ yields $\varphi(0)=R \circ \varphi \circ R(0)=R(b)=\frac{b}{|b|^{2}}$. Recall now that $\mathcal{M}_{n}\left(B_{1}, B_{1}\right)$ is the group of isometries of $\left(B_{1}, d\right)$, where $d$ denotes the hyperbolic metric of $B_{1}$ (see [1, Chapter 3]). Thus, by triangle inequality,

$$
d(\varphi(x), 0) \leq d(\varphi(x), \varphi(0))+d(\varphi(0), 0)=d(x, 0)+d\left(\frac{b}{|b|^{2}}, 0\right) .
$$

The hyperbolic distance is related with the Euclidean distance by the formula $d(x, 0)=\log \left(\frac{1+|x|}{1-|x|}\right)$. Therefore we deduce from (2.2) that if $x \in s B_{1}$ and $\varphi \in$ $\mathcal{M}_{n}^{E}\left(B_{1}, B_{1}\right),|\varphi(x)|$ satisfies the inequality

$$
\frac{|\varphi(x)|+1}{|\varphi(x)|-1} \leq \frac{(s+1)\left(h_{0}+1\right)}{(s-1)\left(h_{0}-1\right)} .
$$

Hence $h_{1}$ in the claim (3) of the lemma is implicitly defined by

$$
\frac{h_{1}+1}{h_{1}-1} \leq \frac{(s+1)\left(h_{0}+1\right)}{(s-1)\left(h_{0}-1\right)} \text {. }
$$

The assertion $s \varphi(B) \subset \varphi\left(h_{1} B\right)$. is equivalent to $\varphi^{-1}(s B) \subset h_{1} B$ and hence it follows from the above reasoning.

The following proposition relies on the fact that $\mathcal{M}_{n}$ is a finite dimensional manifold and $\mathcal{M}_{n}(h B)$ is a compact manifold. Thus all metrics are equivalent.

Proposition 2.2 ([21, Chapter 4. Lemma 2.5]). Let $B=B(a, r)$ and $\varphi, \psi \in$ $\mathcal{M}_{n}(h B)$ with $h>1$. Then there exists a constant $C_{4}=C_{4}(h)$ such that for any $x, y \in B$ we have that

$$
|D \varphi(x)-D \psi(x)| \leq \frac{C_{4}}{|B|} \int_{B}|D \varphi-D \psi|
$$

and,

$$
|D \varphi(x)-D \varphi(y)| \leq \frac{1}{r} C_{4} \max _{B}|D \varphi||x-y| .
$$


The next lemma states that derivatives of Möbius transforms are like constants in the following sense: If they are sufficiently close in a ball, they are also close in appropriate dilations of this ball.

Lemma 2.3 ([21, Chapter 4. Lemma 4.1]). Let $\varphi \in \mathcal{M}_{n}$ such that the inequality

$$
|\varphi(x)-x| \leq r \epsilon
$$

holds for all $x \in B(a, r)$, where $\epsilon>0$. Let $h>0$. Then there exist constants $\alpha=\alpha(h)$ and $C_{5}=C_{5}(h)$ such that if $\epsilon<\alpha$ then the inequality

$$
|D \varphi(x)-I| \leq C_{5} \epsilon
$$

holds for all $x \in h B$.

We conclude the section by introducing the elliptic equations needed in Section 4 and DeGiorgi-Nash's Theorem on the regularity of the solutions.

Definition 2.4. Let $F: \mathbb{R}^{n} \rightarrow \mathbb{R}$ be a convex function such that $|F(A)| \leq C(1+$ $\left.|A|^{p}\right)$. Then a mapping $z \in W^{1, p}\left(\Omega, \mathbb{R}^{n}\right) ; z=\left(z_{1}, \ldots, z_{n}\right)$ is said to be $F$ harmonic in $\Omega$ if each of the coordinates $z_{i}$ satisfies that

$$
\operatorname{div}\left(D F\left(D z_{i}\right)\right)=0 \text { in } \Omega
$$

Equivalently, $z_{i}$ minimizes $\int_{\Omega} F(D v)$ respect to its own boundary values.

Proposition 2.5 ([4, 10]). Let $z$ be an $F$-harmonic mapping in $B$. Let $F: \mathbb{R}^{n} \rightarrow \mathbb{R}$ be a $C^{\infty}$ uniformly convex and such that $|D F(A)| \leq C_{F}|A|,\left|D^{2} F\right| \leq C_{F}$ for $C_{F}>0$. Let $0 \leq h<1$. Then, $z \in C^{\infty}(h B)$ and there exists a number $0<\alpha<1$, $\alpha=\alpha(h, F)$ and a constant $C_{6}=C_{6}(h, F)$ such that

$$
[D z]_{C^{\alpha}(h B)} \leq C_{6}\left(\int_{B}|D z|^{2}\right)^{\frac{1}{2}}
$$

\section{3. $\mathcal{M}_{n}^{E}$ as a subset of $\mathcal{M}_{n}$}

In this section we prove Theorem 1.4 for $v \in \mathcal{M}_{n}(\Omega) \backslash \mathcal{M}_{n}^{E}\left(\Omega^{\prime}\right)$. Firstly, we reduce the situation to the case where $E$ is connected in Proposition 3.1. The essential point in the proof is Proposition 2.2 stating that if $\varphi \in \mathcal{M}_{n}^{E^{\prime}}$ then $D \varphi$ is Lipschitz and the Lipschitz constant depends only on $E^{\prime}$. Thus, there is no loss of generality assuming $E=E^{m \cdot M}$. We treat this case in Proposition 3.2. The essential observation for the proof of Proposition 3.2 is that if $\varphi \notin \mathcal{M}_{n}^{E}\left(\Omega^{\prime}\right)$, then there exists a ball $\tilde{B} \subset \Omega \backslash \Omega^{\prime}$ with $|\tilde{B}|^{\frac{1}{n}} \approx \operatorname{dist}\left(\Omega^{\prime}, \partial \Omega\right)$ such that for $x \in \tilde{B}, D \varphi(x) \notin E$. This, and that for 
$\varphi \in \mathcal{M}_{n}^{E}$ the oscillation of $|D \varphi|$ is uniformly controlled, provides us a constant $C=C\left(\Omega^{\prime}, E\right)$ such that

$$
\min \left\{\int_{\Omega^{\prime}} \operatorname{dist}_{M S O(n)}^{2}(D \varphi), \int_{\Omega^{\prime}} \operatorname{dist}_{m S O(n)}^{2}(D \varphi)\right\} \leq C \int_{\tilde{B}} \operatorname{dist}_{E}^{2}(D \varphi) .
$$

Therefore, we can conclude by means of Theorem 1.1 .

If one tries to follow a similar scheme for proving an estimate up to the boundary one faces the situation of the Example 3.3 presented at the end of the section.

Proposition 3.1. Let $E, \Omega^{\prime}, \Omega$ as in Theorem 1.4 and let $\left\{E_{i}\right\}_{i=1}^{I}$ be the connected components of $E$. Then, there exists $C_{7}=C_{7}\left(E, \Omega^{\prime}, \Omega\right)$ such that for every $\varphi \in$ $\mathcal{M}_{n}(\Omega)$

$$
\min _{i}\left\{\int_{\Omega^{\prime}} \operatorname{dist}_{E_{i}}^{2}(D \varphi)\right\} \leq C_{7} \int_{\Omega} \operatorname{dist}_{\cup_{i} E_{i}}^{2}(D \varphi) .
$$

Proof. By induction, it is enough to prove the theorem for $E$ having two connected components $E=E_{1} \cup E_{2}$. Let $m_{1}=\min _{E_{1}}|A| \leq \max _{E_{1}}|A|=m_{2}<\min _{E_{2}}|A|=$ $M_{1} \leq \max _{E_{2}}|A|=M_{2}$ and $\rho=M_{1}-m_{2}=\operatorname{dist}\left(E_{1}, E_{2}\right)$ (Here we use the invariance of $E$ under $\mathrm{SO}(\mathrm{n})$ ). We firstly observe that there exist constants $c_{1}=$ $c_{1}(E), c_{2}=c_{2}(E)$ such that if either $|A| \geq 2 M_{2}$ or $|A| \leq \frac{1}{2 m_{1}}$ then

$$
\operatorname{dist}_{E_{i}}(A) \leq c_{1} \operatorname{dist}_{E}(A)
$$

and $\operatorname{dist}_{E}(A) \geq c_{2}$.

Let $\Omega^{E}=\left\{x \in \Omega^{\prime}: \frac{1}{2 m_{1}} \leq|D \varphi(x)| \leq 2 M_{2}\right\}$. Suppose that $\left|\Omega^{E}\right| \leq \frac{1}{2}|\Omega|$. Then we have that $2\left|\Omega \backslash \Omega^{E}\right| \geq|\Omega|$ and therefore

$$
\begin{aligned}
\int_{\Omega} \operatorname{dist}_{E_{1}}^{2}(D \varphi) & \leq c_{1} \int_{\Omega \backslash \Omega^{E}} \operatorname{dist}_{E}^{2}(D \varphi) d x+4 M_{2}^{2}|\Omega| \\
& \leq c_{1} \int_{\Omega \backslash \Omega^{E}} \operatorname{dist}_{E}^{2}(D \varphi) d x+\frac{8 M_{2}^{2}}{c_{2}} c_{2}\left|\Omega \backslash \Omega^{E}\right| \\
& \leq c_{3} \int_{\Omega \backslash \Omega^{E}} \operatorname{dist}_{E}^{2}(D \varphi),
\end{aligned}
$$

and (3.1) holds. Hence, we can assume that $\left|\Omega^{E}\right| \geq \frac{1}{2}|\Omega|$.

Now, if

$$
\operatorname{dist}_{E_{1}}(D \varphi(x)) \geq \frac{\rho}{2},
$$

for every $x \in \Omega_{E}$ it would follow that

$$
\operatorname{dist}_{E_{2}}(D \varphi(x)) \leq c_{4} \operatorname{dist}_{E}(D \varphi)
$$


with $c_{4}=\frac{4}{\rho} M_{2}$. Together with (3.2) we would have that

$$
\operatorname{dist}_{E_{2}}(D \varphi(x)) \leq \max \left\{c_{1}, c_{4}\right\} \operatorname{dist}_{E}(D \varphi)
$$

for all $x \in \Omega$ and (3.1) would be trivial.

Since the same argument works if we exchange $E_{1}$ and $E_{2}$ we are left to the case where there exist two points $x_{1}, x_{2} \in \Omega^{E}$ such that

$$
\begin{aligned}
& \operatorname{dist}_{E}\left(D \varphi\left(x_{1}\right)\right)=\operatorname{dist}_{E_{1}}\left(D \varphi\left(x_{1}\right)\right) \leq \frac{\rho}{2}, \\
& \operatorname{dist}_{E}\left(D \varphi\left(x_{2}\right)\right)=\operatorname{dist}_{E_{2}}\left(D \varphi\left(x_{2}\right)\right) \leq \frac{\rho}{2} .
\end{aligned}
$$

Then, since $\Omega$ is connected we can assume $\Omega^{\prime}$ to be connected. Thus, there exists $x_{0} \in \Omega^{\prime}$ with $\left|D \varphi\left(x_{0}\right)\right|=\frac{M+m}{2}$ and consequently $\operatorname{dist}_{E_{i}}\left(D \varphi\left(x_{0}\right)\right)=\frac{\rho}{2}$ for $i=$ 1,2 . Since $\Omega^{\prime} \Subset \Omega$, there is no loss of generality assuming the existence of $r_{1}=$ $r_{1}\left(\Omega^{\prime}, \Omega\right)$ such that $B\left(x_{0}, r_{1}\right) \subset \Omega^{\prime}$. Furthermore, since $2\left|\Omega^{E}\right| \geq|\Omega|$ and $\Omega$ is bounded there exists $r_{2}=r_{2}(\Omega, n)$ such that $B\left(x_{0}, r_{2}\right) \subset \Omega^{E}$.

Now it follows from Proposition 2.2 that $D \varphi_{\mid B\left(x_{0}, r_{2}\right)}$ is Lipschitz with a constant $L\left(E, r_{2}\right)$. Thus, there is new $r_{3}=r_{3}\left(\Omega, \Omega^{\prime}, E\right)$ such that on $B\left(x_{0}, r_{3}\right)$, $\operatorname{dist}_{E}(D \varphi(x)) \geq \frac{\rho}{4}$. But now this implies that

$$
\begin{aligned}
\int_{\Omega} \operatorname{dist}_{E}^{2}(D \varphi) & =\int_{\Omega \backslash \Omega^{E}} \operatorname{dist}_{E}^{2}(D \varphi)+\int_{\Omega^{E}} \operatorname{dist}_{E}^{2}(D \varphi) \\
& \geq \int_{\Omega \backslash \Omega^{E}} \operatorname{dist}_{E}^{2}(D \varphi)+\int_{B\left(x_{0}, r_{3}\right)} \operatorname{dist}_{E}^{2}(D \varphi) \\
& \geq \int_{\Omega \backslash \Omega^{E}} \operatorname{dist}_{E}^{2}(D \varphi) d x+c_{5} \rho^{2} r_{3}^{n} \geq c_{6} \int_{\Omega^{\prime}} \operatorname{dist}_{E_{1}}^{2}(D \varphi),
\end{aligned}
$$

where $c_{6}=\max \left\{\frac{c_{5} \rho^{2} r_{3}^{n}}{M_{2}^{2}\left|\Omega^{\prime}\right|}, c_{1}^{2}\right\}$. The proof is concluded.

Proposition 3.2. Let $\Omega^{\prime}, \Omega$ and $E$ as in Theorem 1.4. Let $E \subset E^{\prime}$. Then, there exists a constant $C_{8}=C_{8}\left(\Omega, \Omega^{\prime}, E^{\prime}\right)$ such that for every $\varphi \in \mathcal{M}_{n}^{E^{\prime}}(\Omega)$ there exists $\varphi_{\Omega^{\prime}} \in \mathcal{M}_{n}^{E}\left(\Omega^{\prime}\right)$ satisfying

$$
\int_{\Omega^{\prime}}\left|D \varphi-D \varphi_{\Omega^{\prime}}\right|^{2} \leq C_{8} \int_{\Omega} \operatorname{dist}_{E}^{2}(D \varphi) .
$$

Proof. By Theorem 1.1 and Proposition 3.1 it suffices to prove the thesis for

$$
E=E^{m, M}
$$

$0<m<M<\infty$. 
Let $\varphi \in \mathcal{M}_{n}^{E^{\prime}}(\Omega)$ and suppose that $\varphi_{\mid \Omega^{\prime}} \notin \mathcal{M}_{n}^{E}\left(\Omega^{\prime}\right)$. Then either $\max _{\Omega^{\prime}}|D \varphi|>$ $M$ or $\min _{\Omega^{\prime}}|D \varphi|<m$. We consider the two possibilities separately.

Case $\max _{\Omega^{\prime}}|D \varphi|>M$.

Let $d=\operatorname{dist}\left(\Omega^{\prime}, \partial \Omega\right)$. We will deduce (3.3) from the next two claims:

There exists $c_{1}=c_{1}\left(\Omega, \Omega^{\prime}, E\right)$ such that

$$
\left(\max _{\Omega_{\frac{d}{2}}^{\prime}}|D \varphi|-M\right)^{2} \leq c_{1} \int_{\Omega} \operatorname{dist}_{E}^{2}(D \varphi) .
$$

There exists $c_{2}=c_{2}\left(\Omega, \Omega^{\prime}\right)$ such that if $\min _{\Omega^{\prime}}|D \varphi|<M$ we have that

$$
\left(\max _{\Omega_{\frac{d}{2}}^{\prime}}|D \varphi|-M\right) \geq c_{2}\left(M-\min _{\Omega^{\prime}}|D \varphi|\right) .
$$

Let us supposed we have proved (3.4) and (3.5) and deduce the thesis from them. Indeed, (3.4) and (3.5) imply that if $x \in \Omega^{\prime}$ and $|D \varphi(x)| \leq M$, then it holds that

$$
\operatorname{dist}_{M \operatorname{SO}(n)}^{2}(D \varphi(x)) \leq c_{3} \int_{\Omega} \operatorname{dist}_{E}^{2}(D \varphi),
$$

with $c_{3}=\frac{c_{1}}{c_{2}^{2}}$. On the other hand if $|D \varphi(x)| \geq M$, then $\operatorname{dist}_{M \operatorname{SO}(n)}^{2}(D \varphi(x))=$ $\operatorname{dist}_{E}^{2}(D \varphi(x))$. Hence,

$$
\int_{\Omega^{\prime}} \operatorname{dist}_{M \operatorname{SO}(n)}^{2}(D \varphi) \leq c_{3} \int_{\Omega} \operatorname{dist}_{E}^{2}(D \varphi)
$$

and thus (3.3) would follow from Theorem 1.1 with $\varphi_{\Omega^{\prime}}=A x$ where $A \in M \operatorname{SO}(n)$.

To prove the claims (3.4) and (3.5) we need further notation. Since $\varphi \in$ $\mathcal{M}_{n}^{E^{\prime}}(\Omega)$ we have that $|D \varphi(x)|=r^{2}|x-a|^{-2}$ where $r \in \mathbb{R}$ and $a \in \mathbb{R}^{n} \backslash \Omega$. Let $x_{0} \in \Omega^{\prime}$ be such that $\left|D \varphi\left(x_{0}\right)\right|=\max _{\Omega^{\prime}}|D \varphi|>M$ and let $L(t): \mathbb{R} \rightarrow \mathbb{R}^{n}$ be defined by $L(t)=x_{0}+t \frac{a-x_{0}}{\left|a-x_{0}\right|}$. By triangle inequality if $x \in B\left(L\left(\frac{d}{4}\right), \frac{d}{4}\right)$ then $|D \varphi(x)| \geq M$. Thus for such an $x$

$$
\operatorname{dist}_{E}(D \varphi(x))=|D \varphi(x)|-M=\operatorname{dist}_{M \operatorname{SO}(n)}(D \varphi(x)) .
$$

Therefore we can apply Theorem 1.1 to $\varphi$ in $B\left(L\left(\frac{d}{4}\right), \frac{d}{4}\right)$. Together with (3.7) it yields an $R_{\varphi} \in \mathrm{SO}(n)$ such that

$$
\int_{B\left(L\left(\frac{d}{4}\right), \frac{d}{4}\right)}\left|D \varphi-M R_{\varphi}\right|^{2} \leq c_{4} \int_{B\left(L\left(\frac{d}{4}\right), \frac{d}{4}\right)} \operatorname{dist}_{E}^{2}(D \varphi) .
$$

Since $\varphi \in \mathcal{M}_{n}(\Omega), \varphi \in \mathcal{M}_{n}\left(B\left(L\left(\frac{d}{4}, 4 \frac{d}{4}\right)\right)\right.$. Thus, we can apply Proposition 2.2 to obtain

$$
\left\|D \varphi-M R_{\varphi}\right\|_{L^{\infty}\left(B\left(L\left(\frac{d}{4}\right), \frac{d}{4}\right)\right)} \leq \frac{C_{4}(4)}{\left|B\left(L\left(\frac{d}{4}\right), \frac{d}{4}\right)\right|} \int_{B\left(L\left(\frac{d}{4}\right), \frac{d}{4}\right)}\left|D \varphi-M R_{\varphi}\right| .
$$


Combining (3.8), (3.9) and triangle inequality yields

$$
\left(\left|D \varphi\left(L\left(\frac{d}{2}\right)\right)\right|-\left|M R_{\varphi}\right|\right)^{2} \leq c_{1} \int_{\Omega} \operatorname{dist}_{E}^{2}(D \varphi),
$$

with $c_{1}=\frac{c_{4} C_{4}(4)}{\left|B\left(L\left(\frac{d}{4}\right), \frac{d}{4}\right)\right|}$. We have obtained the desired (3.4).

For (3.5), we observe that the function $f(t)=|D \varphi(L(t))|$ is convex and increasing for $t<d$. Let $\left|D \varphi\left(x_{1}\right)\right|=\min _{\Omega^{\prime}}|D \varphi(x)|$. Then $\left|D \varphi\left(x_{1}\right)\right|=f\left(t_{1}\right)$ where $t_{1}=\left|x_{1}-a\right|-\left|a-x_{0}\right|$. Since $f(0)>M, t_{1}<0$ and by triangle inequality $-t_{1} \leq \operatorname{diam}\left(\Omega^{\prime}\right)$. Now since $f$ is convex it holds that

$$
f\left(\frac{d}{2}\right)-f(0) \geq \frac{d}{-2 t_{1}}\left(f(0)-f\left(t_{1}\right)\right) .
$$

Replacing $f$ by its values, we see that (3.11) is indeed (3.5) with $c_{2}=\frac{d}{2 \operatorname{diam}\left(\Omega^{\prime}\right)}$. Therefore the proof is concluded in the case $\max _{\Omega^{\prime}}|D \varphi|>M$.

Case $\min _{\Omega^{\prime}}|D \varphi|<m$.

By considering the inverse mapping $\varphi^{-1}$ we will reduce the situation to the case $\max _{\Omega^{\prime}}|D \varphi| \geq M$. Since $\varphi \in \mathcal{M}_{n}^{E^{\prime}}(\Omega)$ we can apply Proposition 2.1 to find a constant $s=s\left(E^{\prime}, \Omega^{\prime}, \Omega\right)$ such that $\operatorname{dist}\left(\varphi\left(\Omega^{\prime}\right), \partial \varphi(\Omega)\right) \geq s$. Hence we can argue as in the previous case with $\varphi^{-1}$ in the place of $\varphi$ and $\frac{1}{m}$ in the place of $M$. We obtain that if $\inf _{\Omega^{\prime}}|D \varphi(x)|<m$, there exists $c_{5}=c_{5}(E, s), R \in \mathrm{SO}(n)$ and $B \subset \varphi(\Omega) \backslash \varphi\left(\Omega^{\prime}\right)$ with $|B| \geq c_{6} s^{n}$ such

$$
\int_{\varphi\left(\Omega^{\prime}\right)}\left|\frac{1}{m} R-D \varphi^{-1}\right|^{2} \leq c_{5} \int_{B} \operatorname{dist}_{\frac{1}{m} \operatorname{SO}(n)}^{2}\left(D \varphi^{-1}\right)
$$

and for all $x \in B\left|D \varphi^{-1}(x)\right| \geq \frac{1}{m}$. Now recall that if $\varphi \in \mathcal{M}_{n}^{E^{\prime}}(\Omega) m^{n} \leq J_{\varphi} \leq M^{n}$ and that for $\rho \in \mathbb{R} \operatorname{dist}_{\rho \mathrm{SO}(n)}(D \varphi(x))=|\rho-| D \varphi(x)||$. Therefore we can change variables in (3.12) to obtain that

$$
\int_{\Omega^{\prime}}\left|m R^{-1}-D \varphi\right|^{2} \leq c_{7} \int_{\varphi^{-1}(B)} \operatorname{dist}_{m \operatorname{SO}(n)}^{2}(D \varphi)
$$

with $c_{7}=c_{7}\left(E, E^{\prime}, \Omega^{\prime}, \Omega\right)$. We also used that $|A-B|=|A||B|\left|A^{-1}-B^{-1}\right|$. Finally for $x \in \varphi^{-1}(B)|D \varphi(x)|<m$ and hence $\operatorname{dist}_{m S O(n)}^{2}(D \varphi(x))=\operatorname{dist}_{E}^{2}(D \varphi(x))$. Thus, the desired estimate

$$
\int_{\Omega^{\prime}}\left|m R^{-1}-D \varphi\right|^{2} \leq c_{7} \int_{\varphi^{-1}(B)} \operatorname{dist}_{E}^{2}(D \varphi)
$$

follows and the proof is concluded. 
In the following example $E=E^{m, M}$.

Example 3.3. There exists a sequence $\varphi_{j} \in \mathcal{M}_{n}\left(B_{1}\right)$ such that

$$
\lim _{j \rightarrow \infty} \frac{\inf _{\psi \in \mathcal{M}_{n}^{E}\left(B_{1}\right)} \int_{B_{1}}\left|D \varphi_{j}-D \psi\right|^{2}}{\int_{B_{1}} \operatorname{dist}_{E}^{2}\left(D \varphi_{j}\right)}=\infty .
$$

Proof. Let $\varphi=r \frac{x-\lambda e_{1}}{\left|x-\lambda e_{1}\right|^{2}}$ with $\lambda$ and $r$ chosen so that $\left|D \varphi\left(e_{1}\right)\right|=M$ and $\left|D \varphi\left(-e_{1}\right)\right|=m$ where $e_{1}=(1,0 \ldots, 0)$. Let us consider the sequence $\varphi_{j}=$ $r \frac{x-\left(\lambda-\frac{1}{j}\right) e_{1}}{\left|x-\left(\lambda-\frac{1}{j}\right) e_{1}\right|^{2}}$ and set $t_{j}=\left|D \varphi_{j}\left(e_{1}\right)\right|-M$. On one hand, we have that

$$
\begin{aligned}
\int_{B_{1}} \operatorname{dist}_{E}^{2}\left(D \varphi_{j}\right) & \leq t_{j}^{2}\left|\left\{x \in B_{1}: \operatorname{dist}_{E}^{2}\left(D \varphi_{j}\right)>0\right\}\right| \\
& =t_{j}^{2}\left|\left\{x \in B_{1}:\left|D \varphi_{j}\right| \geq M\right\}\right| .
\end{aligned}
$$

On the other hand, let $\psi \in \mathcal{M}_{n}^{E}\left(B_{1}\right)$ and $\varphi_{j}$ defined as above with $\frac{1}{j} \leq \frac{\lambda-1}{2}$. Then $\varphi_{j}$ and $\psi \in \mathcal{M}_{n}(h B)$ for some $h>1, h$ not depending of $j$. Thus, Proposition 2.2 yields a constant $c_{1}=C_{4}(h)$ such that

$$
\begin{aligned}
t_{j}^{2}=\left(\left|D \varphi_{j}\left(e_{1}\right)\right|-M\right)^{2} & \leq\left|D \varphi_{j}\left(e_{1}\right)-D \psi\left(e_{1}\right)\right|^{2} \\
& \leq c_{1} \int_{B_{1}}\left|D \varphi_{j}-D \psi\right|^{2} .
\end{aligned}
$$

If we put (3.15) and (3.16) together, we obtain that

$$
\frac{\inf _{\psi \in \mathcal{M}_{n}^{E}\left(B_{1}\right)} \int_{B_{1}}\left|D \varphi_{j}-D \psi\right|^{2}}{\int_{B_{1}} \operatorname{dist}_{E}^{2}\left(D \varphi_{j}\right)} \geq \frac{c_{1}}{\left|x \in B_{1}:\right| D \varphi_{j}|\geq M|},
$$

which proves the claim.

\section{Proof of Theorem 1.4 in a ball}

The starting point for the proof of Theorem 1.1 in [7] is that thanks to the structure of the set $\mathrm{SO}(n)$ it suffices to prove Theorem 1.1 for mappings whose components are uniformly Lipschitz harmonic functions. We start along the same lines than in [7] since $E$ enjoys a nice structure as well. 


\subsection{Reduction to Lipschitz mappings}

Proposition 4.1. Let $E \subset E^{m, M}$. There exists constants $C_{9}=C_{9}(n), C_{10}=$ $C_{10}(M)$ such that if $v \in W^{1,2}(B)$ there exists $v_{M} \in W^{1, \infty}(\Omega)$ such that

- $\left\|D v_{M}\right\|_{L^{\infty}(B)} \leq C_{9} M$ and

- $\int_{B}\left|D v-D v_{M}\right|^{2} \leq C_{10} \int_{B} \operatorname{dist}_{E}^{2}(D v)$.

Proof. The appendix A.1 in [7] yields $v_{M}$ satisfying

- $\left\|D v_{M}\right\|_{L^{\infty}(B)} \leq c_{1} M$

- $\int_{B}\left|D v-D v_{M}\right|^{2} \leq c_{2} \int_{\{x \in B:|D v| \geq 2 M\}}|D v|^{2}$.

Since if $|A| \geq 2 M,|A| \leq 2 \operatorname{dist}_{E}(A)$ we are done.

\subsection{Reduction to $F$-harmonic mappings}

As $\mathrm{SO}(n)$ is related to harmonic functions so is $\mathrm{CO}_{+}(n)$ to $n$-harmonic functions. However since the $n$-harmonic equation is degenerate the arguments below applied to $n$-harmonic functions would yield a wrong exponent in (4.7). Instead we take advantage of $E$ being a compact subset of $\mathrm{CO}_{+}(n)$ bounded away from 0 . This allows us to modify $|\cdot|^{n}$ near 0 and $\infty$ constructing a function $F$ such that $F(A)=$ $|A|^{n}$ for matrices $A \in E$ but with the right growth at 0 and $\infty$. In this way, the set $E$ is related to minimizers of a variational problem $\int_{\Omega} F(D v)$, where $F$ has the appropriate growth.

Throughout the section the $n$-tuple of $n$-vectors $\left(A_{1}, A_{2}, \ldots, A_{n}\right)$ stands for the matrix with rows $\left(A_{1}, A_{2}, \ldots, A_{n}\right)$ and the cofactor matrix of $A,(\operatorname{Cof}(A))_{i j}=$ $\partial_{i j} \operatorname{det}(A)$.

Lemma 4.2. There exists a function $\psi \in C^{\infty}(\mathbb{R}, \mathbb{R})$ such that the function $F(A)$ : $\mathbb{R}^{n} \rightarrow \mathbb{R}$ defined by $F(A)=\psi(|A|)$ belongs to $C^{\infty}\left(\mathbb{R}^{n}, \mathbb{R}\right)$ and satisfies that $D F(A) \leq C_{F}(1+|A|)$ and $\frac{1}{C_{F}} \leq D^{2} F(A) \leq C_{F}$ with $C_{F}=C_{F}(E)$. Moreover, for every $A \in E$ it holds that $\left(D F\left(A_{1}\right), D F\left(A_{i}\right), D F\left(A_{n}\right)\right)=\operatorname{Cof}(A)$. Equivalently $\operatorname{Cof}(A)=D \tilde{F}$ where $\tilde{F}(A)=\sum_{i=1}^{n} F\left(A_{i}\right)$.

Proof. Let $F(A)=\psi(|A|)$. Then a direct calculation gives that

$$
\begin{aligned}
D F(A)=\psi^{\prime}(|A|) \frac{A}{|A|}, \quad D^{2} F(A)= & \psi^{\prime \prime}(|A|)\left(\frac{A}{|A|} \otimes \frac{A}{|A|}\right) \\
& +\frac{\psi^{\prime}(|A|)}{|A|}\left(I-\frac{A}{|A|} \otimes \frac{A}{|A|}\right) .
\end{aligned}
$$

If we put

$$
\psi_{1}(x)=n(n-1)\left(\int_{0}^{x} \int_{0}^{y} z^{n-2} \chi_{(0, M)}(z)+\left(1-\chi_{(0, M)}(z)\right) M^{n-2} d z d y\right),
$$


$\psi_{1}(x)=x^{n} \chi_{0, M}+\left(\left(1-\chi_{(0, M)}(x)\right) a x^{2}+b x+c\right.$ with $a, b \geq 0$ and it satisfies the claims of the Lemma 4.2 except in a neighborhood of the origin. Then we consider $\psi_{2}(x)=\max \left\{\left(\frac{m_{1}}{2}\right)^{n-2} x^{2}, \psi_{1}(x)\right\}$. This new function satisfies the claims but it is not smooth. We repair this by replacing $\psi_{2}$ by a smooth approximation of it in a neighborhood of $\frac{m_{1}}{2}$. Let $r$ and $\epsilon$ be small numbers, and $\varphi \in C_{0}^{\infty}\left(\frac{m_{1}}{2}-2 r, \frac{m_{1}}{2}+2 r\right)$ $\varphi=1$ on $\left(\frac{m_{1}}{2}-r, \frac{m_{1}}{2}+r\right)$ and let $\rho_{\epsilon}$ be an approximation of the identity. Then setting

$$
\psi_{\epsilon, r}=\varphi\left(\rho_{\epsilon} * \psi_{2}\right)+(1-\varphi) \psi_{2}
$$

for $\epsilon$ and $r$ small enough it is not hard to check that $F(A)=\psi_{\epsilon, r}(|A|)$ satisfies all the conditions concerning regularity in the statement of the lemma. By (4.1) the bounds for the derivatives of $F$ depend on the bounds for the derivatives of $\varphi_{\epsilon, r}$, which in turn depend on $m$ and $M$. Hence the constant $C_{F}$ depends on the set $E$. Concerning regularity since $\psi \in C^{\infty}$ and is quadratic near 0 the smoothness of $F$ follows.

Finally, if $A=\left(A_{1}, A_{2}, \ldots, A_{n}\right) \in \mathrm{CO}_{+}(n), \operatorname{det}(A)=|A|^{n}=\left|A_{i}\right|^{n}$ where $\left|A_{i}\right|$ is the Euclidean norm of the vector $A_{i}$. Since for $A \in E F\left(A_{i}\right)=\left|A_{i}\right|^{n}$, it holds that $\tilde{F}(A)=n \operatorname{det}(A)$ as well. If we differentiate both sides of this equality we obtain that for $A \in E D \tilde{F}(A)=\left(D F\left(A_{1}\right), D F\left(A_{i}\right), D F\left(A_{n}\right)\right)=\operatorname{Cof}(A)$ as claimed.

$\operatorname{Set} h(A)=D \tilde{F}(A)-\operatorname{Cof}(A)$.

Lemma 4.3. Let $v \in W^{1,2}\left(B, \mathbb{R}^{n}\right)$. Then

$$
v=w+z,
$$

where $z$ is an F-harmonic mapping in $B$ and $w \in W_{0}^{1,2}\left(B, \mathbb{R}^{n}\right)$ is such that

$$
\int_{B}|D w|^{2} \leq C_{F} \int_{B}|h(D v)|^{2} .
$$

Proof. To obtain the decomposition we solve the following system

$$
\begin{cases}\operatorname{Div}(D \tilde{F}(D z))=0 & \text { in } B \\ z=v & \text { on } \partial B .\end{cases}
$$

Here for a matrix valued function $A(x)$ the operator $\operatorname{Div}(A(x))$ yields an $n$-vector with $\operatorname{div}\left(A_{i}(x)\right)$ as components. Thus, given that

$$
D \tilde{F}(D z)=\left(D F\left(D z_{1}\right), D F\left(D z_{2}\right), \ldots, D F\left(D z_{n}\right)\right)
$$

we can express

$$
\operatorname{Div}\left(D \tilde{F}(D z)=\left(\operatorname{div}\left(D z_{1}(x)\right), \cdot \operatorname{div}\left(D_{n}(x)\right)\right)\right.
$$


and solving the system (4.2) is equivalent to solving the $n$ scalar equations

$$
\begin{cases}\operatorname{div}\left(D F\left(D z_{i}\right)\right)=0 & \text { in } B \\ z_{i}=v_{i} & \text { on } \partial B .\end{cases}
$$

In the linear situation occurring in $\mathrm{SO}(n)$ where $F(A)=|A|^{2}$ (4.2) is equivalent to $\operatorname{Div}(D w)=\operatorname{Div}(h(D v))$ for $w=v-z$. In the current nonlinear situation (4.2) means that

$$
\begin{cases}\operatorname{Div}(A(x, D w))=-\operatorname{Div}(h(D v)) & \text { in } B \\ w=0 & \text { on } \partial B\end{cases}
$$

where $A(x, \xi)=D \tilde{F}(D v(x)-\xi)-D \tilde{F}(D v(x))$ is an $\mathcal{A}$-harmonic type of operator. From the uniform convexity of $F$, and the subsequent strong monotonicity of $D F$ it follows that

$$
\int_{B}|D w|^{2} \leq C_{F} \int_{B}\langle D \tilde{F}(D v-D w)-D \tilde{F}(D v), D w\rangle .
$$

Plugging (4.4) into (4.5) and using Hölder's inequality we obtain

$$
\int_{B}|D w|^{2} \leq C_{F} \int_{B}|h(D v)|^{2}
$$

as desired.

Remark 4.4. Let $v$ be $C M$-Lipschitz. Since $h$ is uniformly Lipschitz in $B(0,2 M)$, we have that $|h(D v)| \leq c_{1} \operatorname{dist}_{E}(D v)$ and therefore Lemma 4.3 reads as

$$
\int_{B}|D w|^{2} \leq C_{11} \int_{B}\left|\operatorname{dist}_{E}(D v)\right|^{2} .
$$

In addition,

$$
\int_{B}|D z|^{2} \leq \int_{B}|D v|^{2}+\left|\operatorname{dist}_{E}(D v)\right|^{2}
$$

Now if $v$ is $C_{9} M$-Lipschitz $\operatorname{dist}_{E}(D v) \leq c_{2}(M+m)$ and thus

$$
\int_{B}|D z|^{2} \leq C_{12},
$$

with $C_{11}=C_{11}(M), C_{12}=C_{12}(M)$. 


\subsection{Compactness}

In the case of $\mathrm{SO}(n)$ it is proved in [7] the existence of a constant $C$ such that for any harmonic function $z$, there is $R \in \mathrm{SO}(n)$ such that

$$
\|R-D z\|_{L^{\infty}(h B)} \leq C\left(\int_{B} \operatorname{dist}_{\mathrm{SO}(n)}^{2}(D z)\right)^{\frac{1}{4}},
$$

for $h<1$. In our setting, we do not know how to obtain this type of explicit estimate. However, the regularity of $F$-harmonic functions allows to use a compactness argument to prove an expression like (4.8), where $\left(\int_{B} \operatorname{dist}_{\mathrm{SO}(n)}^{2}(D z)\right)^{\frac{1}{4}}$ is replaced by $\rho_{1}\left(\int_{B} \operatorname{dist}_{E}^{2}(D z)\right)$. Here, $\rho_{1}(\epsilon)$ is an unknown increasing function that at least converges to 0 as $\epsilon$ goes to 0 .

Lemma 4.5. Let $h<1$ and $B_{1}$ the unit ball. There exists a function $\rho_{1}(\epsilon)$ with $\lim _{\epsilon \rightarrow 0} \rho_{1}(\epsilon)=0$ such that for every $F$-harmonic mapping $z$ there exists, $\varphi \in$ $\mathcal{M}_{n}^{E}\left(B_{1}\right)$ satisfying

$$
\|D \varphi-D z\|_{L^{\infty}\left(h B_{1}\right)} \leq \rho_{1}\left(\int_{B_{1}} \operatorname{dist}_{E}^{2}(D z)\right) .
$$

Proof. Let

$$
\begin{gathered}
\delta(z)=\inf _{\varphi \in \mathcal{M}_{n}^{E}\left(B_{1}\right)}\|D \varphi-D z\|_{L^{\infty}\left(h B_{1}\right)} \text {, and } \\
\rho_{1}(\epsilon)=\sup \left\{\delta(z): z F \text {-harmonic, } \int_{B_{1}} \operatorname{dist}_{E}^{2}(D z) \leq \epsilon\right\} .
\end{gathered}
$$

Clearly $\rho_{1}$ is a positive bounded decreasing function whence $\lim _{\epsilon \rightarrow 0} \rho_{1}(\epsilon)=\eta$ exists. Let us argue by contradiction by assuming that $\eta>0$. This means that there exists a sequence of $F$-harmonic mappings $\left\{z_{j}\right\}$ such that $\delta\left(z_{j}\right) \geq \frac{\eta}{2}$. But

$$
\int_{B_{1}} \operatorname{dist}_{E}^{2}\left(D z_{j}\right) \leq \frac{1}{j}
$$

i.e. $\lim _{j \rightarrow 0} \int_{B_{1}} \operatorname{dist}_{E}^{2}\left(D z_{j}\right)=0$. Since $E$ is a compact set it follows that $\int_{B_{1}}\left|D z_{j}\right|^{2} \leq c_{1}, c_{1}=c_{1}(E)$. Therefore there is no loss of generality assuming that $D z_{j} \rightarrow D \varphi$ weakly in $L^{2}\left(B_{1}\right)$ for some $\varphi \in W^{1,2}(\Omega)$. Furthermore, by Proposition 2.5 and Ascoli-Arzela theorem $D z_{j}$ converge to $D \varphi$ uniformly on compact subsets of $B_{1}$. This together with (4.10) this yields that $D \varphi \in E$ a.e.x. Given that $E$ is bounded, $D \varphi \in W^{1, \infty}\left(B_{1}\right)$ and therefore, by Theorem 1.3, $\varphi \in \mathcal{M}_{n}^{E}\left(B_{1}\right)$. Finally, $D z_{j} \rightarrow D \varphi$ uniformly in $h B_{1}$, which contradicts that $\eta>0$ and proves the claim. 


\subsection{Linearization and local estimate}

We start this section by recalling how the geometry of $\mathrm{CO}_{+}(n)$ and of $\mathcal{M}_{n}$ are related (a detailed account of this is given in [21]). We denote the tangent plane to $\mathrm{CO}_{+}(n)$ at the identity matrix by $\mathrm{TCO}_{+}(\mathrm{n})$. It can be seen that

$$
A \in \mathrm{TCO}_{+}(\mathrm{n}) \Longleftrightarrow \frac{A+A^{t}}{2}=\frac{1}{n} \operatorname{Tr}(A) I .
$$

Thus,

$$
\Theta(A)=\left|\frac{A+A^{t}}{2}-\frac{1}{n} \operatorname{Tr}(A) I\right|
$$

is equivalent to the distance from $A$ to $\mathrm{TCO}_{+}(\mathrm{n})$. It can be shown from the definition of $\Theta$ that the set of functions $\left\{u: D u \in \mathrm{TCO}_{+}(\mathrm{n})\right\}$ is a finite dimensional vector space of dimension $d=\frac{(n+2)(n+1)}{2}$. We denote it by $\Sigma_{n}$. Now recall that $\mathcal{M}_{n}$ is a Lie group isomorphic to $\mathrm{SO}_{o}(n+2,1)$, the connected component of the classical group $\mathrm{SO}(n+2,1)$ containing the identity. Alternatively, one can arrive to $\Sigma_{n}$ as the Lie Algebra of $\mathcal{M}_{n}$ isomorphic to so $(n+2,1)$ (The Lie algebra of $\mathrm{SO}_{o}(n+$ $2,1)$ ). Let $\left\{u_{i}\right\}_{i=1}^{d}$ be a basis of $\Sigma_{n}$. Then we consider the projection $\Pi_{\Sigma_{n}, B_{1}}$ : $W^{1, p}\left(B_{1}\right) \rightarrow \Sigma_{n}$ defined by

$$
f \rightarrow \sum_{i=1}^{d} u_{i} \frac{1}{\left|B_{1}\right|} \int_{B_{1}}\left\langle f, u_{i}\right\rangle .
$$

We will need the following Korn type of inequality first proved by Reshetnyak [20]. We refer to [21] for an extended discussion about this type of linear operators.

Theorem 4.6 ([21, Chapter 3, Theorem 3.2]). Let $1<p<\infty$. Then There exists a constant $C_{13}=C_{13}(p)$ such that for every function $g \in W^{1, p}\left(B, \mathbb{R}^{n}\right)$ we have that

$$
\left\|D(g)-D \Pi_{\Sigma_{n}}(g)\right\|_{L^{p}(B)} \leq C_{13}\|\Theta(D(g))\|_{L^{p}(B)} .
$$

The following proposition and corollary originate from Reshetnyak's study of the stability of the Möbius group in terms of quasiconformality when the distortion is close to 1 [21]. It is based on the following consideration: If a mapping $g$ happens to satisfy that $\Pi_{\Sigma_{n}}(g)=0$, then Theorem 4.6 implies that $\|D(g)\|_{L^{p}(B)} \leq$ $\|\Theta(D(g))\|_{L^{p}(B)}$. We would like to apply this argument to the mapping $f-x$. Of course, $\Pi_{\Sigma_{n}}(f-x)$, does not need to be 0 . In Proposition 4.7 we use an homotopy argument to prove that if there exists $\varphi$ so that $\Pi_{\Sigma_{n}}(f \circ \varphi-x)=0$. Let us remark that for this it is not needed to control $D f-I$. However to replace $\operatorname{dist}_{\mathrm{TCO}_{+}(\mathrm{n})}(A-I)$ by $\operatorname{dist}_{\mathrm{CO}_{+}(n)}(A)$, it is needed that $D A-I$ is small, and hence we need to have control over $D(f \circ \varphi)-I$. It turns out that to achieve this estimate is rather involved in the quasiconformal setting [21] but it is much simpler in 
our situation. This is due to the fact that the modulus of continuity of $D f$ can be assumed to be bounded independently of $f$.

In the Corollary 4.8 we use the Taylor expansion of $\operatorname{dist}_{\mathrm{CO}_{+}(n)}^{2}$ to express the local estimate in the form needed for the next section.

Proposition 4.7. Let $0 \leq h \leq 1, \gamma_{1}, \gamma_{2}$ positive constants, $B=B(a, r)$ an arbitrary ball and $f \in C^{1, \alpha}((1+\bar{h}) B)$ such that

$$
[D f]_{C^{\alpha}((1+h) B)} \leq \gamma_{1} r^{-\alpha} .
$$

Then there exists a positive number $\delta_{1}\left(h, \gamma_{2}\right)$, a non increasing function $\rho_{2}(\epsilon)$ with $\lim _{\epsilon \rightarrow 0} \rho_{2}(\epsilon)=0$ and a constant $C_{14}$ such that, if

$$
\|D f-I\|_{L^{\infty}((1+h) B)} \leq \epsilon \leq \delta_{1}
$$

then, there exists $\varphi \in \mathcal{M}_{n}(2 B)$ satisfying the next three properties:

1. $\|D(f \circ \varphi-x)\|_{L^{2}(B)} \leq C_{14}\|\Theta(D(f \circ \varphi-x))\|_{L^{2}(B)}$,

2. $\|D(f \circ \varphi-x)\|_{L^{\infty}(B)} \leq \rho_{2}(\epsilon)$ and

3. $\|\varphi-x\|_{W^{1, \infty}(2 B)} \leq r \gamma_{2}$.

The function $\rho_{2}$ depends on the modulus of continuity of $D f$ and $C_{14}=C_{13}(2)$, where $C_{13}$ was introduced in Theorem 4.6.

Proof. Since both the assumptions and the claim depend on $D f$, there is no loss of generality assuming that $f(0)=0$. We can also assume that $B=B_{1}$. The case of an arbitrary ball follows by composing with similarities.

For every continuous mapping we define $\Lambda_{f}: \Sigma_{n} \rightarrow \Sigma_{n}$ by $u \rightarrow \Pi_{\Sigma_{n}}(f \circ$ $\left.\varphi_{u}-x\right)$ where $\Pi_{\Sigma_{n}}=\Pi_{\Sigma_{n}, B_{1}}$ is the projection from $W^{1, p}\left(B_{1}, \mathbf{R}^{n}\right) \rightarrow \Sigma_{n}$ defined in (4.11) and $\varphi_{u}=\exp (u)$. Here exp is the exponential mapping from $\Sigma_{n}$ to $\mathcal{M}_{n}$ (Observe that using the isomorphism between $\mathcal{M}_{n}$ and $\mathrm{SO}_{o}(n+2,1)$ it is not hard to obtain an explicit expression of exp. Denoting by $F$ the isomorphism between $\mathcal{M}_{n}$ and $\mathrm{SO}_{o}(n+2,1), \exp (u)=F^{-1}(\exp (d F(u))$, where exp is the matrix exponential in $\left.M^{n+2 \times n+2}\right)$. The following properties of the exponential will be useful:

a) Let us declare $M(s)=\max \left\{\left\|\varphi_{u}(x)-x\right\|_{W^{1, \infty}(2 B)}: u \in B(0, s) \subset \Sigma_{n}\right\}$. Then, since all the topologies in $\mathcal{M}_{n}$ are the same,

$$
\lim _{s \rightarrow 0} M(s)=0 .
$$

b) Let us state $\Lambda=\Lambda_{I}$. One of the properties defining the exponential mapping is that $\frac{d}{d t} \varphi_{t u}(x)_{\mid 0}=u(x)$. It is easy to see that this implies that for any

$$
h \in \Sigma_{n}, \quad \Lambda^{\prime}(0) h=h .
$$

Hence, the inverse function theorem tells us that there exists $s_{0}>0$ such that $\Lambda$ maps $B\left(0, s_{0}\right)$ homeomorphically into $\Sigma_{n}$. In particular, $\operatorname{deg}_{\Lambda}\left(0, B\left(0, s_{0}\right)\right)$, the topological degree of $\Lambda$ at zero in $B\left(0, s_{0}\right)$, is 1 . 
Due to (4.12) we can assume without loss of generality that

$$
M\left(s_{0}\right) \leq \gamma_{2} .
$$

Let $s \leq s_{0}$, then we have that

$$
\begin{aligned}
\left|\Lambda_{f}(u)-\Lambda(u)\right|_{\mid B(0, s)} & =\left|\Pi_{\Sigma_{n}}\left(f \circ \varphi_{u}-\varphi_{u}\right)\right| \\
& \leq\left\|\Pi_{\Sigma_{n}}\right\|\left\|\varphi_{u} \mid D f-I\right\|_{L^{\infty}((1+h) B)} \leq c_{1} \epsilon,
\end{aligned}
$$

where \|\| stands for the operator norm of $\Pi_{\Sigma_{n}}$ and

$$
c_{1}=\left\|\Pi_{\Sigma_{n}}\right\| \max _{x \in B, u \in B\left(0, s_{0}\right)}\left|\varphi_{u}(x)\right| .
$$

By (4.13) we have that $c_{1} \leq\left\|\Pi_{\Sigma_{n}}\right\|\left(1+M\left(s_{0}\right)\right) \leq\left\|\Pi_{\Sigma_{n}}\right\|\left(1+\gamma_{2}\right)$.

Let us declare

$$
m(s)=\min _{\partial B(0, s)}|\Lambda(u)| .
$$

Since $\Lambda$ is an homeomorphism in $B\left(0, s_{0}\right)$ and $\Lambda(0)=0$ we have that $0<m(s)$ for every $r<s_{0}$. Moreover, since $\varphi_{u} \rightarrow x$ uniformly

$$
\lim _{s \rightarrow 0} m(s)=0 .
$$

Then we define $s_{\epsilon}$ by

$$
s_{\epsilon}=\inf \left\{s: m(s) \geq c_{1} \epsilon+\epsilon\right\},
$$

where $c_{1}$ is defined in (4.15). Then, (4.16) implies that

$$
\lim _{\epsilon \rightarrow 0} s_{\epsilon} \rightarrow 0 \text {. }
$$

Now, the definition of $s_{\epsilon}$ and (4.14) yield that if $s_{\epsilon} \leq s_{0}$, then

$$
t \Lambda+(1-t) \Lambda_{f}(u) \neq 0 \text { for } u \in \partial B\left(0, s_{\epsilon}\right) .
$$

Thus, $\operatorname{deg}\left(0, B\left(0, s_{\epsilon}\right)\right)$ remains constant through the homotopy (see for example [11, chapter 14]).

Let us declare $\delta_{1}=\frac{m\left(s_{0}\right)}{c_{1}+1}$. If $\epsilon<\delta_{1}$, then $s_{\epsilon}<s_{0}$ and thus, $\operatorname{deg}_{\Lambda_{f}}\left(0, B\left(0, s_{\epsilon}\right)\right)=$ $\operatorname{deg}_{\Lambda}\left(0, B\left(0, s_{\epsilon}\right)\right)=1$. Therefore, there exists $u \in B\left(0, s_{\epsilon}\right)$ such that $\Lambda_{f}(u)=0$. When we apply Theorem 4.6 to $f \circ \varphi_{u}-x$, (1) follows.

To prove (2) we observe that

$$
\begin{aligned}
\left|D\left(f \circ \varphi_{u}(x)\right)-I\right| \leq|D f(x)-I| & +|D f(x)|\left|D \varphi_{u}(x)-I\right| \\
& +\left|D \varphi_{u}(x)\right|\left|D f\left(\varphi_{u}(x)\right)-D f(x)\right| .
\end{aligned}
$$

By definition we have that $|D f(x)-I| \leq \epsilon$, that $\left|D \varphi_{u}(x)-I\right| \leq M\left(s_{\epsilon}\right)$ and that $\left|\varphi_{u}(x)-x\right| \leq M\left(s_{\epsilon}\right)$. Hence, using the Hölder regularity of $D f$ we obtain that

$$
\left|D\left(f \circ \varphi_{u}(x)-x\right)\right| \leq \epsilon+(1+\epsilon) M\left(s_{\epsilon}\right)+\gamma_{1}\left(\left(1+M\left(s_{\epsilon}\right)\right) M\left(s_{\epsilon}\right)^{\alpha}\right)=\rho_{2}(\epsilon) .
$$

Finally, (4.17) and (4.12) imply that $\lim _{\epsilon \rightarrow 0} \rho_{2}(\epsilon)=0$ as desired. The proposition is proved since $M\left(s_{0}\right) \leq \gamma_{2}$ by (4.13), which yields (3). 
Corollary 4.8 (Local estimate). Let $0<h \leq 1, \gamma_{1}, \gamma_{2}>0$ and $B(a, r)$ a ball. Then there exists $\delta_{2}=\delta_{2}\left(h, \gamma_{1}, \gamma_{2}, \alpha\right)$ and $C_{15}$ such that if $f$ satisfies

1. $[D f]_{C^{\alpha}((1+h) B)} \leq \gamma_{1} r^{-\alpha}$ and

2. $\|D f-I\|_{L^{\infty}((1+h) B)} \leq \delta$,

then there exists $\varphi \in \mathcal{M}_{n}(2 B)$ with $\|\varphi(x)-x\|_{W^{1, \infty}(2 B)} \leq r \gamma_{2}$ and

$$
\int_{B}|D(f \circ \varphi)-I|^{2} \leq C_{15} \int_{B} \operatorname{dist}_{\mathrm{CO}_{+}(n)}^{2}(D(f \circ \varphi)) .
$$

Proof. The corollary is proved by plugging the results from the previous proposition into the Taylor expansion of dist $\mathrm{CO}_{+}(n)$. By Taylor we have that

$$
\left|\operatorname{dist}_{\mathrm{CO}_{+}(n)}^{2}(A)\right|=c_{1}|\Theta(A-I)|^{2}+O\left(|A-I|^{4}\right) .
$$

Therefore,

$$
|\Theta(A-I)|^{2} \leq c_{2}\left|\operatorname{dist}_{\mathrm{CO}_{+}(n)}^{2}(A)\right|+O\left(|A-I|^{4}\right) .
$$

We first require that $\delta_{2} \leq \delta_{1}$ to apply Proposition 4.7 to the mapping $f$ and find the desired $\varphi$. Using Proposition 4.7 (1), putting $A=D(f \circ \varphi)$ in (4.19), and rearranging the resulting expression yield

$$
\begin{aligned}
\int_{B}|D(f \circ \varphi)-I|^{2} & \leq c_{3} \int_{B} \operatorname{dist}_{\mathrm{CO}_{+}(n)}^{2}(D(f \circ \varphi)) \\
& +c_{4} \int_{B}|D(f \circ \varphi)-I|^{4} .
\end{aligned}
$$

We finally impose that $\delta$ also satisfies $c_{4} \rho_{2}^{4}(\delta) \leq \frac{1}{2} \rho_{2}^{2}(\delta)$. Then Proposition 4.7 (2) implies that $c_{4}|D(f \circ \varphi)-I|^{4} \leq \frac{1}{2}|D(z \circ \varphi)-I|^{2}$. Hence, we can absorb $c_{4} \int_{B}|D(f \circ \varphi)-I|^{4}$ to the left hand side to conclude that

$$
\int_{B}|D(f \circ \varphi-I)|^{2} \leq c_{5} \int_{B} \operatorname{dist}_{\mathrm{CO}_{+}(n)}^{2}(D(f \circ \varphi)),
$$

as desired.

\subsection{Theorem 1.4 in a ball}

In this section we put together all the information obtained before to obtain the Theorem 1.4 in a ball. We state it as a separate proposition for later uses. 
Proposition 4.9. Let $E \subset \mathrm{CO}_{+}(n)$ be compact, finitely connected with

$$
\operatorname{SO}(n) E=E
$$

and $0 \notin E$. Let $B$ a ball. Then there exists a constant $C_{16}=C_{16}(E)$ such that for any $v \in W^{1,2}\left(4 B, \mathbb{R}^{n}\right)$ there exists $\varphi \in \mathcal{M}_{n}^{E}\left(B, \mathbb{R}^{n}\right)$ such that

$$
\int_{B}|D \varphi-D v|^{2} \leq C_{16} \int_{4 B} \operatorname{dist}_{E}^{2}(D v) .
$$

The sketch of the proof is the following: Let

$$
\epsilon=\int_{4 B} \operatorname{dist}_{E}^{2}(D v)
$$

Using Subsections 4.1, 4.2 one finds that it is enough to prove the proposition for $z$ an $F$-harmonic mapping such that $D z$ is uniformly Hölder in $B^{\prime} \subset 4 B$. Next section 4.3 yields $\varphi_{1} \in \mathcal{M}_{n}^{E}(4 B)$ such that

$$
\left\|D \varphi_{1}-D z\right\|_{L^{\infty}\left(B^{\prime}\right)} \leq \rho_{1}(\epsilon) .
$$

Then, changing variables with $\varphi_{1}$ we discover that the mapping $f=z \circ \varphi_{1}^{-1}$ fulfills the condition required in Corollary 4.8, the local estimate, in a ball $B^{\prime \prime} \subset \varphi_{1}\left(B^{\prime}\right)$. Therefore Corollary 4.8 yields $\varphi_{2}$ such that

$$
\int_{B^{\prime \prime \prime}}\left|D\left(z \circ \varphi_{1}^{-1} \circ \varphi_{2}\right)-I\right|^{2} \leq C \int_{B^{\prime \prime \prime}} \operatorname{dist}_{\mathrm{CO}_{+}(n)}^{2}\left(D z \circ \varphi_{1}^{-1} \circ \varphi_{2}\right) .
$$

where $B^{\prime \prime \prime} \subset B^{\prime \prime}$. Setting $\varphi_{3}=\varphi_{2}^{-1} \circ \varphi_{1}$, we can change variables by $\varphi_{3}$ finding that

$$
\int_{2 B}\left|D z-D \varphi_{3}\right|^{2} \leq C \int_{4 B} \operatorname{dist}_{\mathrm{CO}_{+}(n)}^{2}(D z) .
$$

where $2 B \subset \varphi_{3}^{-1}\left(B^{\prime \prime \prime}\right)$. The existence of the balls $B^{\prime}, B^{\prime \prime}, B^{\prime \prime \prime}$ fulfilling the properties used above is essentially a consequence of the Lemma 2.1.

Finally, since $\varphi_{3}$ might be in $\mathcal{M}_{n}(2 B) \backslash \mathcal{M}_{n}^{E}(B)$ we use Proposition 3.2 to find $\varphi_{4} \in \mathcal{M}_{n}^{E}(B)$ such that

$$
\int_{B}\left|D \varphi_{3}-D \varphi_{4}\right|^{2} \leq C \int_{4 B} \operatorname{dist}_{\mathrm{CO}_{+}(n)}^{2}\left(D \varphi_{3}\right) .
$$

From here, the proof is concluded by reiterative use of triangle inequality.

We now give the detailed argument.

Proof of Proposition 4.9. By a change of variables with a similarity it is enough to prove the estimate in $B_{1} \subset 4 B_{1}$. We start by using the Lemma 2.1 to select 
the appropriate balls. Let $0<h_{0}, h_{1}, h_{2}<1$ be such for any $\varphi \in \mathcal{M}_{n}^{E}(4 B)$ the following inclusions hold:

$$
\begin{aligned}
\varphi(2 B) \subset h_{0} \varphi(4 B), \quad & \left(h_{0}+3 h_{1}\right) \varphi(4 B) \subset \varphi(4 B) \text { and } \\
& \left(h_{0}+2 h_{1}\right) \varphi(4 B) \subset \varphi\left(h_{2} 4 B\right) .
\end{aligned}
$$

Next, we reduce to a smooth mapping. Firstly we apply Proposition 4.1 to $v$ in $4 B$ to obtain a $C_{9} M$ Lipschitz mapping $v_{M}$. We prove Proposition 4.9 for $v_{M}$. By the Lipschitz bound we have that

$$
\int_{B}\left|D v_{M}-m\right|^{2} \leq c_{1},
$$

$c_{1}=c_{1}(E)$. Hence we can assume that

$$
\int_{4 B} \operatorname{dist}_{E}\left(D v_{M}\right)^{2} \leq \epsilon_{0},
$$

for $0<\epsilon_{0}$ to be determined later. For mappings $v_{M}$ such that (4.26) does not hold, (4.22) is fulfilled with $\varphi(x)=m x$ and $C_{16}=\frac{1}{\epsilon_{0}} c_{1}$.

Let $z$ be the $F$-harmonic mapping obtained by applying Lemma 4.3 to $v_{M}$ in $4 B$. Since $\int_{4 B}|D z|^{2} \leq C_{12}$ by Remark 4.4, Proposition 2.5 yields that for any $h<1$

$$
[D z]_{C^{\alpha}(h 4 B)} \leq c_{2},
$$

with $c_{2}=c_{2}\left(C_{6}, C_{12}, h\right)$. Furthermore, the inequality $\operatorname{dist}_{E}^{2}\left(M_{1}\right) \leq c_{3}\left(\operatorname{dist}_{E}^{2}\left(M_{1}+\right.\right.$ $\left.\left.M_{2}\right)+\left|M_{2}\right|^{2}\right),(4.7)$ and (4.26) imply that

$$
\int_{4 B} \operatorname{dist}_{E}^{2}(D z) \leq c_{4} \epsilon_{0} .
$$

Next, we apply Lemma 4.5 to find the existence of $\varphi_{1} \in \mathcal{M}_{n}^{E}(4 B)$ such that

$$
\left\|D z-D \varphi_{1}\right\|_{L^{\infty}\left(h_{2} 4 B\right)} \leq \rho_{1}(\epsilon),
$$

where $\epsilon=\int_{4 B} \operatorname{dist}_{E}^{2}(D z)$.

We want to translate these estimates to the domain $\varphi_{1}(4 B)$. It follows from Lemma 2.1 that

$$
c_{5}<\left|\varphi_{1}(4 B)\right| \leq c_{6},
$$

$c_{5}=c_{5}(E), c_{6}=c_{6}(E)$. Define $r_{1}>0$ by,

$$
|B(0,1)| r_{1}^{n}=c_{6}
$$

to guarantee that $\operatorname{diam}\left(\varphi_{1}(4 B)\right) \leq 2 r_{1}$. 
Using that $\varphi \in \mathcal{M}_{n}^{E}(4 B)$ we deduce

$$
\begin{aligned}
\left\|D\left(z \circ \varphi_{1}^{-1}\right)-I\right\|_{L^{\infty}\left(\varphi_{1}\left(h_{2} 4 B\right)\right)} & \\
& \leq\left\|D \varphi_{1}^{-1}\right\|_{L^{\infty}\left(\left(\varphi_{1}\left(h_{2} 4 B\right)\right)\right.}\left\|D z-D \varphi_{1}\right\|_{L^{\infty}\left(h_{2} 4 B\right)} \\
& \leq \frac{1}{m} \rho_{1}(\epsilon) .
\end{aligned}
$$

In addition, by (4.27) we have

$$
\begin{aligned}
C^{\alpha}\left(\varphi_{1}\left(h_{2} 4 B\right)\right) & \leq\left\|D \varphi_{1}^{-1}\right\|_{L^{\infty}\left(\left(\varphi_{1}\left(h_{2} 4 B\right)\right)\right.}[D z]_{C^{\alpha}\left(h_{2} 4 B\right)} \\
& \leq \frac{c_{7}}{m}
\end{aligned}
$$

with $c_{7}=c_{7}\left(h_{2}, M\right)$. Furthermore, by Lemma 2.1, Proposition 2.2 and (4.29), $D \varphi_{1}^{-1}$ is uniformly continuous in $\varphi_{1}\left(h_{2} 4 B\right)$. Hence, there exists $c_{8}=c_{8}\left(h_{2}, E\right)$ such that

$$
\left[D\left(z \circ \varphi_{1}^{-1}\right)\right]_{\left.C^{\alpha}\left(\varphi_{1}\left(h_{2} 4 B\right)\right)\right)} \leq c_{8} \leq c_{9} r_{1}^{-\alpha}
$$

by (4.30).

Since by (4.25) $\left.\left(h_{0}+2 h_{1}\right) \varphi_{1}(4 B) \subset \varphi_{1}\left(h_{2} 4 B\right)\right)$, we intend to apply Corollary 4.8 with $B=\left(h_{0}+h_{1}\right) \varphi_{1}(4 B), f=z \circ \varphi_{1}, h=h_{1} \gamma_{1}=c_{9}$ and $\gamma_{2}=\frac{h_{1}}{r_{1}}$. Now, by (4.26) we can impose for $\epsilon_{0}$ that $\rho_{1}\left(\epsilon_{0}\right) \leq m \delta\left(E, h_{1}\right)$. Thus, (4.31) and (4.32) imply that all the requirements of Corollary 4.8 are fulfilled. Hence, we are provided with $\varphi_{2} \in \mathcal{M}_{n}$ such that

$$
\begin{aligned}
\int_{\left(h_{0}+h_{1}\right) \varphi_{1}(4 B)} \mid & D\left(z \circ \varphi_{1}^{-1} \circ \varphi_{2}\right)-\left.I\right|^{2} \\
\leq & c_{10} \int_{\left(h_{0}+h_{1}\right) \varphi_{1}(4 B)} \operatorname{dist}_{\mathrm{CO}_{+}(n)}^{2}\left(D z \circ \varphi_{1}^{-1} \circ \varphi_{2}\right) .
\end{aligned}
$$

with $c_{10}=C_{15}$. Let $\varphi_{3}=\varphi_{2}^{-1} \circ \varphi_{1}$. By Proposition 4.7 (3) and our choice of $\gamma_{2}$ we have

$$
\left\|\varphi_{2}-x\right\|_{W^{1, \infty}\left(\left(h_{0}+h_{1}\right) \varphi_{1}(4 B)\right)} \leq h_{1} .
$$

In addition, $\varphi_{1} \in \mathcal{M}_{n}^{E}\left(h_{2} 4 B\right)$. It follows that $\varphi_{3}^{-1}=\varphi_{1}^{-1} \circ \varphi_{2} \in \mathcal{M}_{n}^{E^{\prime}}\left(\left(h_{0}+\right.\right.$ $\left.h_{1}\right) \varphi(4 B)$ ) for some $E^{\prime}=E^{\prime}(E)$. Therefore, there exist constants $c_{11}=c_{11}(E), c_{12}=$ $c_{12}(E)$ such that

$$
\begin{aligned}
c_{11} J_{\varphi_{3}^{-1}}(x) & =c_{11}\left|D \varphi_{3}^{-1}(x)\right|^{n} \leq\left|D \varphi_{3}^{-1}(x)\right|^{2} \\
& \leq c_{12}\left|D \varphi_{3}^{-1}(x)\right|^{n}=c_{12} J_{\varphi_{3}^{-1}}(x) .
\end{aligned}
$$


Hence if we change variables in (4.33) by $\varphi_{3}^{-1}$ we obtain

$$
\int_{\varphi_{3}^{-1}\left(\left(h_{0}+h_{1}\right) \varphi_{1}(4 B)\right)}\left|D z-D \varphi_{3}\right|^{2} \leq c_{13} \int_{\varphi_{3}^{-1}\left(\left(h_{0}+h_{1}\right) \varphi_{1}(4 B)\right)} \operatorname{dist}_{\mathrm{CO}_{+}(n)}^{2}(D z)
$$

where $c_{13}=c\left(c_{11}, c_{12}\right)$. Now we investigate what is $\varphi_{3}^{-1}\left(\left(h_{0}+h_{1}\right) \varphi_{1}(4 B)\right)$. By (4.34) we have that

$$
h_{0} \varphi_{1}(4 B) \subset \varphi_{2}\left(\left(h_{0}+h_{1}\right) \varphi_{1}(4 B)\right) \subset\left(h_{0}+2 h_{1}\right) \varphi_{1}(4 B) .
$$

On the other hand, $\varphi_{1} \in \mathcal{M}_{n}^{E}(4 B)$ so (4.25) implies that

$$
2 B \subset \varphi_{1}^{-1}\left(h_{0} \varphi_{1}(4 B)\right) \subset \varphi_{3}^{-1}\left(\left(h_{0}+h_{1}\right) \varphi(4 B)\right) \subset 4 B .
$$

Thus,

$$
\int_{2 B}\left|D z-D \varphi_{3}\right|^{2} \leq c_{13} \int_{4 B} \operatorname{dist}_{\mathrm{CO}_{+}(n)}^{2}(D(z))
$$

as desired.

The last obstruction is that $\varphi_{3}=\varphi_{2}^{-1} \circ \varphi_{1}$ is not necessarily in $\mathcal{M}_{n}^{E}(2 B)$ due to $\varphi_{2}^{-1}$. However (4.34) implies that $\varphi_{3} \in \mathcal{M}_{n}^{E^{\prime}}(2 B)$ for $E^{\prime}$ depending on $E$. Since $\operatorname{dist}_{E}^{2}\left(D \varphi_{3}\right) \leq \operatorname{dist}_{E}^{2}(D z)+\left|D z-D \varphi_{3}\right|^{2}$ we can apply Lemma 3.2 to find $\varphi \in \mathcal{M}_{n}^{E}(B)$ such that

$$
\begin{aligned}
\int_{B}|D \varphi-D z|^{2} & \leq \int_{B}\left|D \varphi-D \varphi_{3}\right|^{2}+\left|D z-D \varphi_{3}\right|^{2} \\
& \leq c_{14} \int_{4 B} \operatorname{dist}_{E}^{2}(D z)
\end{aligned}
$$

where $c_{14}=2 c_{13}+C_{8}$.

Lastly, we translate the estimate from $z$ to $v$. We have that,

$$
\begin{aligned}
\int_{B}\left|D v-D \varphi_{4}\right|^{2} \leq & C \int_{B}\left|D v-D v_{M}\right|^{2} \\
& +\int_{B}\left|D v_{M}-D z\right|^{2}+\int_{B}\left|D z-D \varphi_{4}\right|^{2} .
\end{aligned}
$$

Therefore, using again that $\operatorname{dist}_{E}^{2}(A) \leq \operatorname{dist}_{E}^{2}(B)+|A-B|^{2}$ for $A, B \in M^{n \times n}$ together with Proposition 4.1, Remark 4.4 and the estimate (4.36) we can conclude that

$$
\int_{B}\left|D v-D \varphi_{4}\right|^{2} \leq c_{15} \int_{4 B} \operatorname{dist}_{E}^{2}(D v),
$$

where $c_{15}=c_{15}\left(C_{10}, C_{F}, c_{14}\right)$. 


\section{Proof of Theorem 1.4}

The arguments in Section 4 can be modified to be applied to a compact set $\Omega^{\prime}$ instead of a ball, but several technical problems arise. Therefore, we have preferred to keep Section 4 simple and prove Theorem 1.4 for a generic compact set by a covering argument. The idea is as follows: We can suppose that the compact set $\Omega^{\prime}$ is a finite union of overlapping balls $\left\{B^{i}\right\}$. Therefore we can apply Proposition 4.9 to each $B^{i}$. This yields a family of Möbius transforms $\left\{\varphi_{i}\right\}$ such that $D \varphi_{i}$ is close to $D v$ in $B^{i}$. This implies that if we take two balls $B^{i}, B^{j} D \varphi_{i}$ is close to $D \varphi_{j}$ in $B^{i} \cap B^{j}$. The fundamental property of Möbius mappings used here is Lemma 2.3. Lemma 2.3 says that if we choose the parameters correctly, $D \varphi_{j}$ is close to $D \varphi_{i}$ not only in $B^{i} \cap B^{j}$ but in the whole of $\Omega^{\prime}$. Therefore if we choose a ball $B^{0}, D \varphi_{0}$ is close to $D \varphi_{i}$ for any $B^{i}$ such that $B^{i}$ intersects $B^{0}$. Moreover, given an arbitrary ball $B^{\prime}$ we can link it with $B^{0}$ by a finite chain of pairwise intersecting balls. It is not hard to see that it follows that the Möbius map $\varphi^{\prime}$ corresponding to $B^{\prime}$ is close to $\varphi_{0}$ in $\Omega^{\prime}$ as well. Therefore all the $\varphi_{i}$ are close to each other in $\Omega^{\prime}$ and hence any of them is close to $v$ in the whole $\Omega^{\prime}$. The following proposition shows that this can be made rigorous.

Proposition 5.1. Let $\Omega^{\prime} \Subset \Omega \subset \mathbb{R}^{n}$ with $\Omega$ connected. Then there exists a constant $C_{17}=C_{17}\left(E, \Omega^{\prime}, \Omega\right)$ and $E^{\prime} \subset \mathrm{CO}_{+}(n), E^{\prime}$ depending on $\Omega^{\prime}$ and $E$, such that for any $v \in W^{1,2}\left(\Omega, \mathbb{R}^{n}\right)$ there exists $\varphi \in \mathcal{M}_{n}^{E^{\prime}}(\Omega)$ such that

$$
\int_{\Omega^{\prime}}|D \varphi-D v|^{2} \leq C_{17} \int_{\Omega} \operatorname{dist}_{E}^{2}(D v) .
$$

Proof. Firstly, we observe that it is enough to prove the estimate assuming that

$$
\int_{\Omega} \operatorname{dist}_{E}^{2}(D v) \leq \epsilon_{0}
$$

as in the proof of Proposition 4.9.

Let $r=\operatorname{dist}\left(\Omega^{\prime}, \Omega\right)$. Since $\Omega^{\prime}$ is compact there exists a family of balls $\left\{B\left(x_{i}, \frac{r}{16}\right)\right\}_{i=1}^{n}$ such that $\Omega^{\prime} \subset \cup_{i=1}^{n} B\left(x_{i}, \frac{r}{16}\right)$ with $n=n\left(\Omega^{\prime}\right)$. Since $\Omega^{\prime}$ can be assumed to be connected for each $x_{i}$ there exist $x_{i}^{\prime}, y_{i}$ such that $B\left(y_{i}, \frac{r}{16}\right) \subset B\left(x_{i}, \frac{r}{8}\right) \cap$ $B\left(x_{i}^{\prime}, \frac{r}{8}\right)$. We set $B^{i}=B\left(x_{i}, \frac{r}{8}\right)$. Then $\Omega^{\prime} \subset \cup_{i=1}^{n} B^{i}$ and $8 B^{i} \subset \Omega$. In addition given any two balls $B^{0}, B^{\prime} \in\left\{B^{i}\right\}_{i=1}^{n}$ there exist two chains of balls $\left\{B^{j}\right\}_{j=1}^{m} \subset$ $\left\{B^{i}\right\}_{i=1}^{n}$ and, $\left\{P_{j}\right\}_{j=1}^{m}$ such that $B^{1}=B^{0}, B^{m}=B^{\prime}, 2 P_{j} \subset 2 B^{j} \cap 2 B^{j+1}$ and $\left|P_{j}\right| \geq c r^{n}$.

We apply Proposition 4.22 to $2 B^{0}$ to find the existence of $\varphi_{0} \in \mathcal{M}_{n}^{E}\left(2 B^{0}\right)$ such that

$$
\int_{2 B^{0}}\left|D v-D \varphi_{0}\right|^{2} \leq c_{1} \int_{8 B^{0}} \operatorname{dist}_{E}^{2}(D v)
$$


$c_{1}=C_{16}$. We plan to show that $\varphi_{0} \in \mathcal{M}_{n}^{E}\left(\Omega^{\prime}\right)$ and that

$$
\int_{B^{\prime}}\left|D v-D \varphi_{0}\right|^{2} \leq c_{2} \int_{\Omega} \operatorname{dist}_{E}^{2}(D v) .
$$

Clearly, the thesis follows from (5.3) since $B^{\prime} \in\left\{B^{i}\right\}_{i=1}^{n}$ was arbitrary.

Application of Proposition 4.22 in each of the balls $2 B^{j}$, yields $\varphi_{j} \in \mathcal{M}_{n}^{E}\left(B^{j}\right)$ such that

$$
\int_{2 B^{j}}\left|D v-D \varphi_{j}\right|^{2} \leq \int_{\Omega} \operatorname{dist}_{E}^{2}(D v) .
$$

Then it is easy to see that proving (5.3) is equivalent to proving that

$$
\int_{B^{\prime}}\left|D \varphi_{j}-D \varphi_{j-1}\right|^{2} \leq c_{1} \int_{\Omega} \operatorname{dist}_{E}^{2}(D v)
$$

for every $j=1 \ldots m$.

Set $\theta_{j}=\varphi_{j-1}^{-1} \circ \varphi_{j}$. Then (5.4) and Proposition 2.2 imply that for $x \in P_{j}$

$$
\begin{aligned}
\max _{\Omega^{\prime}}\left\{\left|D \theta_{j}(x)-I\right|,\left|D \theta_{j}^{-1}(x)-I\right|\right\} & \leq c_{3}\left|D \varphi_{j}-D \varphi_{j-1}\right|^{2} \\
& \leq c_{4} \int_{\Omega} \operatorname{dist}_{E}^{2}(D v)
\end{aligned}
$$

where in the first inequality we have used that $\varphi_{j}, \varphi_{j-1} \in \mathcal{M}_{n}^{E}\left(P_{j}\right)$.

Let $h=h\left(\Omega^{\prime}, \Omega\right)$ be such that for every $B, \tilde{B} \in\left\{B_{i}\right\}_{i=1}^{n} 2 B \subset h \tilde{B}$. Let $\alpha(h)$ be given by Lemma 2.3. Then we firstly imposed that $\epsilon_{0} c_{4} \leq \alpha(h)$. Thus, by (5.6) and Lemma 2.3 we have that for $x \in B^{\prime}$

$$
\left|D \theta_{j}(x)-I\right|^{2} \leq c_{5} \int_{\Omega} \operatorname{dist}_{E}^{2}(D v), x \in B^{\prime},
$$

where $c_{5}=C_{5} c_{4}, C_{5}$ being defined in Lemma 2.3. To estimate $\left|D \varphi_{j}-D \varphi_{j-1}\right|$ by $c_{6}\left|D \theta_{j}(x)-I\right|$ with $c_{6}=c_{6}(E)$ it is enough that $\varphi_{j}, \varphi_{j-1} \in \mathcal{M}_{n}^{E^{\prime}}\left(\Omega^{\prime}\right)$ for a set $E^{\prime}$ depending only on $E$. To achieve this we further require that $\epsilon_{0} C_{5} \leq \frac{1}{\operatorname{diam}(\Omega) 2 n}$, This yields,

$$
\max \left\{\left|\varphi_{j-1}^{-1} \circ \varphi_{j}(x)-x\right|,\left|\varphi_{j}^{-1} \circ \varphi_{j-1}(x)-x\right|\right\}<\frac{1}{4 n} .
$$

Therefore, $\left.\left.\varphi_{j}\left(\left(\frac{3}{2}-\frac{1}{4 n}\right) B^{\prime}\right)\right) \subset \varphi_{j-1}\left(\frac{3}{2} B^{\prime}\right) \subset \varphi_{j}\left(\left(\frac{3}{2}+\frac{1}{4 n}\right) B^{\prime}\right)\right)$. Thus, for every $j$ $\varphi_{m}\left(\frac{5}{4} B^{\prime}\right) \subset \varphi_{j}\left(\frac{3}{2}\left(B^{\prime}\right)\right) \subset \varphi_{m}\left(2 B^{\prime}\right)$. In particular this means that $\varphi_{i}$ are finite in $\left(\frac{3}{2} B^{\prime}\right)$. Proposition 2.2 implies that for all $x$ in $B^{\prime}$

$$
\begin{aligned}
\left|D \varphi_{j}(x)\right| \leq \frac{c_{6}}{\left|B^{\prime}\right|} \int_{B^{\prime}}|D \varphi| & \leq c_{6}\left(\frac{\left|\varphi_{j}\left(B^{\prime}\right)\right|}{\left|B^{\prime}\right|}\right)^{\frac{1}{n}} \\
& \leq c_{6}\left(\frac{\left|\varphi_{m}\left(2 B^{\prime}\right)\right|}{\left|B^{\prime}\right|}\right)^{\frac{1}{n}} \leq c_{7} .
\end{aligned}
$$


where $c_{6}=C_{4}, C_{4}$ being from Proposition 2.2 and $c_{7}=2 M c_{6}$. We use here that $\varphi_{m} \in \mathcal{M}_{n}^{E}\left(2 B^{\prime}\right)$. We can argue similarly for $\varphi_{j}^{-1}$ obtaining that $\varphi_{j} \in \mathcal{M}_{n}^{E^{\prime}}\left(B^{\prime}\right)$ as desired. Then, (5.3) follows from (5.7). The Proposition is proved.

Finally, Theorem 1.4 (i) is proved by Proposition 5.1 and Proposition 3.2. Theorem 1.4 (ii) is proved combining Example 3.3 and Theorem 1.1.

\section{References}

[1] A. F. BEARDon, "The Geometry of Discrete Groups", Graduate Texts in Mathematics, Vol. 91, Springer-Verlag, New York, 1995. Corrected reprint of the 1983 original.

[2] N. Chaudhuri and S. MÜlleR, Rigidity estimate for two incompatible wells, Calc. Var. Partial Differential Equations 19 (2004), 379-390.

[3] G. Dal Maso, M. Negri and D. Percivale, Linearized elasticity as $\Gamma$-limit of finite elasticity. Calculus of variations, nonsmooth analysis and related topics, Set-Valued Anal. 10 (2002), 165-183.

[4] E. De Giorgi, Sulla differenziabilità e l'analiticità delle estremali degli integrali multipli regolari, Mem. Accad. Sci. Torino. Cl. Sci. Fis. Mat. Nat. (3) 3 (1957), 25-43.

[5] D. FARACO, Rigidity of sets of matrices, In: "Future Trends in Geometric Function Theory”, Rep. Univ. Jyväskylä Dep. Math. Stat., Vol. 92, Univ. Jyväskylä, Jyväskylä, 2003, $77-83$.

[6] G. Friesecke, R. D. James, M. G. Mora and S. Müller, Derivation of nonlinear bending theory for shells from three-dimensional nonlinear elasticity by Gammaconvergence, C. R. Math. Acad. Sci. Paris 336 (2003), 697-702.

[7] G. Friesecke, S. MÜlleR and R. D. JAmeS, Rigorous derivation of nonlinear plate theory and geometric rigidity, C. R. Math. Acad. Sci. Paris 334 (2002), 173-178.

[8] G. Friesecke, R. D. JAMES and S. MÜlleR, A theorem on geometric rigidity and the derivation of nonlinear plate theory from three-dimensional elasticity, Comm. Pure Appl. Math. 55 (2002), 1461-1506.

[9] F. W. Gehring, Rings and quasiconformal mappings in space, Trans. Amer. Math. Soc. 103 (1962), 353-393.

[10] E. GiUsti, "Direct Methods in the Calculus of Variations", World Scientific Publishing Co. Inc., River Edge, NJ, 2003.

[11] J. Heinonen, T. Kilpeläinen and O. Martio, "Nonlinear Potential Theory of Degenerate Elliptic Equations", Oxford Mathematical Monographs. The Clarendon Press Oxford University Press, New York, 1993. Oxford Science Publications.

[12] S. Hencl, P. Koskela and X. ZHONG, Mappings of finite distortion: Reverse inequalities for the Jacobian, Preprint 310 at the University of Jyväskylä (2004).

[13] T. IWANIEC, p-harmonic tensors and quasiregular mappings, Ann. of Math. (2) 136 (1992), 589-624.

[14] T. IwANIEC and G. MARTin, "Geometric Function Theory and Non-Linear Analysis", Oxford Mathematical Monographs. The Clarendon Press Oxford University Press, New York, 2001.

[15] F. John, Rotation and strain, Comm. Pure Appl. Math. 14 (1961), 391-413.

[16] J. Liouville, Théorème sur l'équation $d x^{2}+d y^{2}+d z^{2}=\lambda\left(d \alpha^{2}+d \beta^{2}+d \gamma^{2}\right.$, J. Math. Pures Appl. 15 (1850).

[17] M. G. MorA and S. MÜlleR, Derivation of the nonlinear bending-torsion theory for inextensible rods by $\Gamma$-convergence, Calc. Var. Partial Differential Equations 18 (2003), 287-305. 
[18] M. G. MorA and S. MÜLleR, A nonlinear model for inextensible rods as a low energy $\Gamma$-limit of three-dimensional nonlinear elasticity, Ann. Inst. H. Poincaré Anal. Non Linéaire 21 (2004), 271-293.

[19] J. G. REŠETNJAK, Liouville's conformal mapping theorem under minimal regularity hypotheses, Sibirsk. Mat. Zh. 8 (1967), 835-840.

[20] J. G. REŠETNJAK, Estimates for certain differential operators with finite-dimensional kernel, Sibirsk. Mat. Zh. 11 (1970), 414-428.

[21] J. G. RešetnJaK, "Stability Theorems in Geometry and Analysis", Mathematics and its Applications, Vol. 304, Kluwer Academic Publishers Group, Dordrecht, 1994. Translated from the 1982 Russian original by N. S. Dairbekov and V. N. Dyatlov, and revised by the author, Translation edited and with a foreword by S. S. Kutateladze.

Departamento de Matematicas Universidad Autónoma de Madrid 28049 Madrid, Spain

daniel.faraco@uam.es

Department of Mathematics and Statistics

University of Jyväskylä

P.O. Box 35 (MaD)

FIN-40014 Jyväskylä, Finland

zhong@maths.jyu.fi 